hep-th/0708.1023

\title{
Quaternionic Kähler Manifolds, Constrained Instantons and the Magic Square: I
}

\author{
Keshav Dasgupta ${ }^{1}$, Véronique Hussin ${ }^{2}$, Alisha Wissanji ${ }^{3}$ \\ 1 Rutherford Physics Building, McGill University, Montréal, QC H3A 2T8, Canada \\ keshav@hep.physics.mcgill.ca \\ 2 Département de Mathématiques et Statistique, UdeM, Montréal, QC H3C 3J\%, Canada \\ hussin@DMS. UMontreal.CA \\ 3 Centre de Recherches Mathématiques, UdeM, Montréal, QC H3C 3J\%, Canada \\ wissanji@crm. umontreal.ca
}

\begin{abstract}
The classification of homogeneous quaternionic manifolds has been done by Alekseevskii, Wolf et al using transitive solvable group of isometries. These manifolds are not generically symmetric, but there is a subset of quaternionic manifolds that are symmetric and Einstein. A further subset of these manifolds are the magic square manifolds. We show that all the symmetric quaternionic manifolds including the magic square can be succinctly classified by constrained instantons. These instantons are mostly semilocal, and their constructions for the magic square can be done from the corresponding Seiberg-Witten curves for certain $\mathcal{N}=2$ gauge theories that are in general not asymptotically free. Using these, we give possible constructions, such as the classical moduli space metrics, of constrained instantons with exceptional global symmetries. We also discuss the possibility of realising the Kähler manifolds in the magic square using other solitonic configurations in the theory, and point out an interesting new sequence of these manifolds in the magic square.
\end{abstract}

August 2007 


\section{Contents}

1. Introduction . . . . . . . . . . . . . . . . . . . . . . . . . . . . . . 1

2. Quaternionic manifolds in string theory . . . . . . . . . . . . . . . . 4

2.1. An example in detail . . . . . . . . . . . . . . . . . . . . . . . . . 5

2.2. Structure of the multiplets . . . . . . . . . . . . . . . . . . . . . . 6

2.3. Few more examples . . . . . . . . . . . . . . . . . . . . . . . . . . 8

3. On the classification of quaternionic manifolds: standard cases . . . . 12

3.1. $S p(n+1)$ quaternionic space . . . . . . . . . . . . . . . . . . . . . 12

3.2. $G_{2}$ quaternionic space . . . . . . . . . . . . . . . . . . . . . . . . . 19

3.2.1. Realisation of the quotient space . . . . . . . . . . . . . . . . . 24

3.2.2. Coordinates of the quotient space . . . . . . . . . . . . . . . . 26

4. On the classification of quaternionic manifolds: the magic square . . . 28

4.1. $E_{6}$ quaternionic space . . . . . . . . . . . . . . . . . . . . . . . . . 32

4.2. $E_{7}$ quaternionic space . . . . . . . . . . . . . . . . . . . . . . . . . 40

4.3. $E_{8}$ quaternionic space . . . . . . . . . . . . . . . . . . . . . . . . . 43

4.4. $F_{4}$ quaternionic space . . . . . . . . . . . . . . . . . . . . . . . . . 45

4.5. Other examples of quaternionic spaces . . . . . . . . . . . . . . . . . . 48

4.5.1. Example 1: $U(p)$ local symmetry and $S U(n+p)$ global symmetry . . 48

4.5.2. Example 2: $S U(2)$ local symmetry and $S O(p+q)$ global symmetry . . 49

4.5.3. Example 3: New sequence of Kahler manifolds in the magic square . . 51

4.6. A note on holomorphic $F$-functions . . . . . . . . . . . . . . . . . . . 53

5. Summary, discussions and future directions . . . . . . . . . . . . . . . 57

\section{Introduction}

A Riemannian manifold $(\mathcal{M}, g)$ is a smooth manifold $\mathcal{M}$ endowed with a metric $g$ defined in $T^{*} \mathcal{M}$. The holonomy of such a connected oriented Riemannian manifold belongs to the following list:

- $S O(n)$ : generic case

- $S U(n), U(n) \subset S O(2 n):$ Calabi-Yau and Kähler cases

- $S p(n), S p(n) \times S p(1) \subset S O(4 n)$ : Hyper-Kähler and Quaternionic Kähler cases

- $G_{2} \subset S O(7), \quad \operatorname{Spin}(7) \subset S O(8)$

The above is the so called Berger's classification theorem [1]. We will be mainly concerned with the following two holonomies: $S p(n)$ and $S p(n) \times S p(1)$. Both these groups act on $\mathbf{H}^{n}=\mathbf{R}^{4 n}$ where $\mathbf{H}^{n}$ is the right vector space over the quaternions $\mathbf{H}$. The $S p(1) \equiv S U(2)$ factor in $S p(n) \times S p(1)$ is the group of unit quaternions acting from the right. 
The quaternionic Kähler manifolds are always Einsteinl for $n \geq 2$ and are self-dual Einstein for $n=1$. They are considered positive if their metrics are complete and have positive scalar curvatures. When the scalar curvatures are zero, then the holonomies of these manifolds reduce to $S p(n)$ and are called the Hyper-Kähler manifolds. Thus clearly quaternionic Kähler manifolds are not Ricci flat.

Examples of quaternionic Kähler manifolds with positive scalar curvatures are given by compact symmetric spaces classified by Wolf [2] and Alekseevski [3] and are known as the Wolf spaces. They are classified by taking centerless Lie group $\mathbf{G}$ which form the isometry group of quaternionic Kähler spaces given as conjugacy classes of $S p(1)$ in $\mathbf{G}$ determined by the highest root of $\mathbf{G}$. These spaces are:

$$
\begin{aligned}
& \mathbf{H} \mathbf{P}^{n}=\frac{S p(n+1)}{S p(n) \times S p(1)}, \quad \mathbf{G r}_{2}\left(\mathbf{C}^{n+2}\right)=\frac{S U(n+2)}{S(U(n) \times U(2))} \\
& \mathbf{G r}_{4}\left(\mathbf{R}^{n+4}\right)=\frac{S O(n+4)}{S O(n) \times S O(4)} \\
& \frac{E_{6}}{S U(6) \times S p(1)}, \quad \frac{E_{7}}{\operatorname{Spin}(12) \times S p(1)}, \quad \frac{E_{8}}{E_{7} \times S p(1)}, \quad \frac{F_{4}}{S p(3) \times S p(1)}, \quad \frac{G_{2}}{S O(4)}
\end{aligned}
$$

Observe that all these spaces are modded by a $S p(1)$ group as expected. This will be useful later when we will map our configurations to semi-local defects.

The above examples are all compact. The non-compact duals are symmetric examples of quaternionic Kähler manifolds with negative scalar curvatures. The non-symmetric, noncompact examples with negative scalar curvatures are also known. However no concrete examples of non-compact non-symmetric positive curvature manifolds are presently known.

In section 2 we will give some examples of symmetric quaternionic Kähler manifolds that appear in string theory. We will study few representative cases - in sections 2.1 and 2.2 - and discuss possible quantum corrections to these spaces. Although most of this is well known, we will present it in a way so as to connect to latter parts of the paper. Important concepts like $c, s$ and $r$-maps will be introduced in section 2.2. The connection between $c$ and $r$-maps, as we will discuss soon, is the following:

$$
\text { Real manifold } \quad \mathrm{r}>\text { Kahler manifold } \longrightarrow \mathrm{c}>\text { Quaternionic Kahler manifold }
$$

which in the language of supergravity means the following: the moduli space of the scalar fields in the vector multiplets for a five dimensional supergravity is a real manifold. Dimensionally reducing this to four dimension yields a Kähler moduli space for the vector

1 By this we mean that the Ricci tensor is proportional to the metric. 
multiplets and further dimensional reduction to three dimensions yields a quaternionic Kähler manifold for the hyper-multiplets. This way of viewing the connection was described by various authors, for example [4], [5], [6] et al, which also led to the connection to the magic square of Freudenthal, Rosenfeld and Tits [7] that we describe at the beginning of section 4 .

Our method of studying the magic square and classifying the quaternionic manifolds is different from what has been attempted so far. We will not analyse using supergravities at all, instead we will describe the whole system via $S U(2)$ gauge theories with global symmetries $\mathcal{G}$ that resemble sectors of $\mathcal{N}=2$ Seiberg-Witten theories [8] in certain parametrisations, but are not asymptotically free. Most of these theories that we analyse are at strong couplings, and in certain cases simple Yang-Mills description may not suffice. Nevertheless we will show that one-instanton moduli spaces could be studied in all these cases, and the corresponding Seiberg-Witten curves could be used to classify the quaternionic spaces. The instantons that we study are not only constrained instantons [9], but are also semilocal [10 2. The Kähler3 and the real spaces could then be classified by other semilocal defects in the theory for certain choices of global symmetries that we analyse using the so-called sequential gauging. These aspects will be described in sections 3 and 4 . In sections 4.1 to 4.4 , we will give strong evidence that all the elements of the magic square [7] can be reproduced starting from certain sectors of $\mathcal{N}=2 S U(2)$ gauge theories with $E_{6}, E_{7}, E_{8}$ and $F_{4}$ global symmetries. The case with $G_{2}$ global symmetry is interesting, and we study this in section 3.2 by detailing an explicit construction of the associated quaternionic space. Normally one wouldn't attach $G_{2}$ to the magic square, but we show that there is a way to incorporate the $G_{2}$ group sequence in the magic square too by adding one extra column.

In section 4.5 we study another example that has not been discussed in the physics literature in details. This new sequencing of the magic square follows rather straightforwardly from our arguments of sequential gauging and could also be added to the magic square by a different choice of the underlying Jordan algebras [12].

In section 4.6 we discuss the sigma model descriptions of these quaternionic spaces by analysing the $F$-functions [5] for all the relevant cases. These $F$-functions are the prepotential that determine the Kähler spaces associated to the quaternionic spaces. We

2 In mathematical terminology therefore these instantons are constrained instanton bundles.

3 These Kähler spaces have been originally classified in [11]. 
then use the $c$-map to determine metrics of all the quaternionic spaces. Finally, in section 5 we conclude with a brief discussion and point out some future directions.

We now begin with the very basics of quaternionic spaces: their role in string theory and gauge theories.

\section{Quaternionic manifolds and string theory}

Our first question would be to ask where does the quaternionic manifolds fit in the whole paradigm of string compactifications. One of the place where these manifolds appear is well known: the moduli space of sigma models for $\mathcal{N}=2$ supergravity in four space-time dimensions. Imposing only global $\mathcal{N}=2$ supersymmetry in four dimensions would lead to sigma models with Hyper-Kähler target spaces [13]. The $\mathcal{N}=2$ multiplets on the other hand can be written in terms of $\mathcal{N}=1$ multiplets. This should tell us the moduli space structure for the corresponding $\mathcal{N}=1$ case also. In fact one can now make the following classifications for $\mathcal{N}=1$ supersymmetry in four dimensions:

- With global supersymmetry the target manifold of a non-linear sigma model can be any Kähler manifold [14].

- With local supersymmetry the target manifold of a non-linear sigma model (which is coupled to supergravity) can only be a restricted Kähler type, also known as a Hodge manifold [15].

The second point is easy to show 15]. We can define a Kähler potential $K$ in terms of the chiral superfield $\Phi^{i}$ and $\bar{\Phi}^{i}$. The terms appearing in the $\mathcal{N}=1$ lagrangian can be expanded from $-3 e^{-\frac{K}{3}}$. The first two relevant terms are

$$
S=\int d^{4} x \sqrt{g}\left[-\frac{R}{2}-g_{i \bar{j}} \partial_{\mu} \phi^{i} \partial^{\mu} \bar{\phi}^{j}+\text { fermions }\right]
$$

where $g_{i \bar{j}}$ (not to be confused with $g$ ) is the metric on the moduli space parametrised by the $\phi^{i}$ - the scalar component in the chiral multiplet $\Phi^{i}$.

The lagrangian (2.1) possesses Kähler invariance under a Kähler transformation. On a local patch it is easy to demonstrate. However to demonstrate this globally one has to show how this transformation can be defined from one patch to another. This gives rise to the consistency condition on triple junctions. From here one can argue the condition required on the elements of the second cohomology group of the target manifold $H^{2}$ : they have to be even integers [15]. Quantization of Newton's constant also follows directly from here [15]. 
On the other hand, the classification for $\mathcal{N}=2$ supersymmetry is more interesting. We discussed this briefly at the beginning of this section. We will now elaborate this in some details. As before, global and local supersymmetry will have distinct properties:

- With global supersymmetry the target manifold of a non-linear sigma model can be any Hyper-Kähler manifold [13]. These are $4 n$ dimensional real Riemannian manifolds with holonomy group lying in $S p(n)$.

- With local supersymmetry the target manifolds of a non-linear sigma model coupled to supergravity can only be quaternionic Kähler manifolds [16]. These manifolds are oriented $4 n$ real dimensional manifolds with holonomy groups lying in $S p(n) \times S p(1)$. These manifolds have negative curvatures given by [16]:

$$
R=-64 \pi n(n+2) G_{N}
$$

where $G_{N}$ is the Newton's constant and $n$ is an integer. This means that the Newton's constant is fixed for a given manifold and not quantised like the earlier $\mathcal{N}=1$ cases. It also means that the global susy couplings are no longer compatible for the local susy case. Only in the limit $G_{N} \rightarrow 0$ the local and global cases could be identified.

\subsection{An example in detail}

Let us consider one concrete example where quaternionic target space can be illustrated. As mentioned above, a sigma model with quaternionic target space has to be coupled to supergravity to make sense. Global supersymmetry cannot yield a quaternionic target space. Therefore our four-dimensional lagrangian can be taken as:

$$
S=\Lambda^{2} \int d^{4} x \sqrt{g}\left[-\frac{R}{2}-\frac{1}{\bar{z}^{f} z^{f}}\left(\partial_{\mu} z^{a}-\frac{z^{a} \bar{z}^{b} \partial_{\mu} z^{b}}{\bar{z}^{c} z^{c}}\right)\left(\partial^{\mu} \bar{z}^{a}-\frac{\bar{z}^{a} z^{d} \partial^{\mu} z^{d}}{\bar{z}^{e} z^{e}}\right)\right]
$$

which is a Fubini-Study metric on the target space. In fact the way we wrote the lagrangian only implies a $\mathbf{C P}^{N}$ target because the coordinates $z^{a}$ go from $a=1$ to $a=N+1$. This is a Kähler metric, but still not quaternionic because the Kähler potential $K$ is

$$
K=\log \left(1+z^{a} \bar{z}^{a}\right)
$$

where $z^{a}$ are summed from $a=1$ to $a=N$ because we are in a patch with $z^{N+1}=1$. To convert (2.3) to quaternionic case, we will first replace all $z^{a} \leftrightarrow q^{a}$, where $q_{a}$ is a $2 \times 2$ matrix given as:

$$
q^{a}=\left(\begin{array}{cc}
q_{0}^{a}+i q_{3}^{a} & q_{2}^{a}+i q_{1}^{a} \\
-q_{2}^{a}+i q_{1}^{a} & q_{0}^{a}-i q_{3}^{a}
\end{array}\right)
$$


where $a=1, \ldots, N$. This would then convert (2.3) to the following quaternionic analogue:

$$
S=\Lambda^{2} \int d^{4} x \sqrt{g}\left[-\frac{R}{2}-\frac{1}{\operatorname{tr}\left(q^{\dagger} \cdot q\right)}\left(\operatorname{tr}\left(\partial_{\mu} q^{\dagger} \cdot \partial^{\mu} q\right)-\frac{\operatorname{tr}\left(q^{\dagger} \cdot \partial_{\mu} q\right) \operatorname{tr}\left(\partial^{\mu} q^{\dagger} \cdot q\right)}{\operatorname{tr}\left(q^{\dagger} \cdot q\right)}\right)\right]
$$

where we have defined $\operatorname{tr}\left(q^{\dagger} \cdot q\right)$ as $\sum_{a} \operatorname{tr}\left(q^{a \dagger} q^{a}\right)$ and similarly the other terms. Such a redefinition to convert (2.3) to (2.6) changes $\mathbf{C P}^{N}$ to $\mathbf{H} \mathbf{P}^{N}$ where

$$
\mathbf{H P}^{N}=\frac{S p(N+1)}{S p(N) \times S p(1)}
$$

The quaternionic analogue of $\mathbf{C} \mathbf{P}^{N}$ i.e $\mathbf{H P}^{N}$ in fact shares the same properties as $\mathbf{C} \mathbf{P}^{N}$ : the $q^{a}$ vectors are defined upto a scaling by a quaternion (recall $z^{a}$ are only defined upto a complex scaling). It is also important to note that any $4 N+3$ sphere is equivalent to a $S^{3}$ fibration over a quaternionic base $\mathbf{H P}^{N}$. This will be useful soon.

\subsection{Structure of the multiplets}

The quaternionic sigma model that we discussed above can be shown to appear in string theory by compactifying Type II strings on a Calabi-Yau three-fold. This leads to $\mathcal{N}=2$ supersymmetry in four dimensional space time with the following generic multiplets:

- Vector multiplet: $\left(A_{\mu}, 2 \phi, 2 \psi\right)$

- Tensor multiplet: $\left(B_{\mu \nu}, 3 \phi, 2 \psi\right)$

- Vector Tensor multiplet: $\left(B_{\mu \nu}, A_{\mu}, \phi, 2 \psi\right)$
- Hypermultiplet: $(4 \phi, 2 \psi)$

- Double Tensor multiplet: $\left(2 B_{\mu \nu}, 2 \phi, 2 \psi\right)$

- Gravity multiplet: $\left(g_{\mu \nu}, A_{\mu}, 2 \psi_{\mu}\right)$ where $\phi$ appearing in all these multiplets are real scalars, $\psi$ are Weyl fermions in four dimensions and $\psi_{\mu}$ are four dimensional gravitinis. Observe that both the double tensor multiplet as well as the tensor multiplet are dual to the hypermultiplet. Similarly the vector tensor multiplet is dual to the vector multiplet. Thus the non-trivial four-dimensional $\mathcal{N}=2$ multiplets are the vector, hyper and the gravity multiplets. Compactifying type IIB theory on a Calabi-Yau three-fold gives rise to the following multiplets:

$$
\left(g_{\mu \nu}, A_{\mu}\right) \oplus h_{12}\left(A_{\mu}, 2 \phi\right) \oplus h_{11}\left(B_{\mu \nu}, 3 \phi\right) \oplus\left(2 B_{\mu \nu}, 2 \phi\right)
$$

where we have ignored the fermionic degrees of freedom. From ten dimensional type IIB point of view, the metric fluctuations give rise to $\left(2 h_{21}+h_{11}\right)$ scalars in four dimensions, the NS and RR antisymmetric tensors both contribute $h_{11}$ scalars in four dimensions along with 
the axio-dilaton contributing two more scalars. Thus the scalars in the vector multiplets all come from the metric fluctuations whereas the scalars in the tensor multiplets come partly from the metric fluctuations and partly from the zero mode fluctuations of the NS and RR two form tensors. Finally the axio-dilaton go to the double tensor multiplet. On the other hand, the vectors in the gravity as well as vector multiplets all come from the zero mode fluctuations of the four-form field. The four-form fluctuations also contribute $h_{11}$ antisymmetric tensors that go to the tensor multiplets whereas the NS and RR two forms both go to the double tensor multiplet. It is also easy to see that once we dualise the tensor and the double tensor multiplets, we will have one gravity multiplet, $h_{12}$ number of vector multiplets and $\left(1+h_{11}\right)$ number of hypermultiplets. On the other hand, type IIA theory when compactified on the same Calabi-Yau will give us the following four-dimensional multiplets:

$$
\left(g_{\mu \nu}, A_{\mu}\right) \oplus h_{11}\left(A_{\mu}, 2 \phi\right) \oplus h_{21}(4 \phi) \oplus\left(B_{\mu \nu}, 3 \phi\right)
$$

where again we have ignored the fermions. To keep track of the scalars: the hypermultiplet scalars come from both the metric fluctuations and a zero mode fluctuations of the threeform field. The vector multiplet scalars come partially from the zero mode fluctuations of the $B_{N S}$ field and partially from the fluctuations of the metric. The dilaton however goes to the tensor multiplet this time. On the other hand, the vectors in the vector multiplets do not come from the IIA vectors but from the zero mode fluctuations of the three form field. In fact the type IIA vector go to the gravity multiplet. The antisymmetric tensor in the tensor multiplet is the type IIA $B_{N S}$ field. Observe also that in the dual picture (i.e dualising the antisymmetric $B_{\mu \nu}$ field) we have one gravity multiplet, $h_{11}$ number of vector multiplets and $\left(1+h_{21}\right)$ number of hypermultiplets. This would be exactly the same as the type IIB multiplets if

$$
h_{11}(\mathrm{IIA})=h_{21}(\mathrm{IIB}), \quad \text { and } \quad h_{21}(\mathrm{IIA})=h_{11}(\mathrm{IIB})
$$

which is of course the statement of mirror symmetry at perturbative tree level.

At this point we should also note that the structures of quaternionic manifolds in string theory are restricted in string compactification. This is easy to see from the fact that some of the scalars in the hypermultiplets come from the zero mode fluctations of the metric. The moduli space of these scalars are Kähler manifolds and therefore the full quaternionic structure of the hypermultiplet moduli space[16], [6] - that come from adding RR scalars to the metric fluctuations - should have a submanifold that is a Kähler 
manifold. This mapping of a Kähler submanifold to the full quaternionic manifold is called as a $c$-mapt[5]. Thus, for example, in type IIB on a Calabi-Yau manifold the quaternionic space is of real dimension $4\left(1+h_{11}\right)$ with a subspace given by

$$
\frac{S U(1,1)}{U(1)} \times \mathcal{M}_{k}
$$

where the first part is parametrised by four-dimensional axion-dilaton i.e the double tensor multiplet, and the second part is the Kähler submanifold. On the other hand, in type IIA theory the first part of (2.11) comes from the four-dimensional tensor multiplet. Thus clearly the hypermultiplet target space cannot be a generic quaternionic manifold because of the $c$-map constraint [5]. Furthermore since the dilaton resides in the hypermultiplets, the tree level picture is not correct. Details of these have been worked out various authors (see for example [17], [18], [19] and references therein). In particular, the perturbative corrections are now fully understood, and not just for the universal hypermultiplet - as shown by [18] there are no quantum corrections beyond 1-loop due to a nonrenormalization theorem. Moreover, the complete worldsheet, $D 1$ and $D(-1)$ instanton corrections in IIB as well as half of the $D 2$ instanton effects in IIA have been determined by [18] together with [19]. The resulting modified moduli spaces are quaternionic in agreement with unbroken $\mathcal{N}=2$ supersymmetry国.

\subsection{Few more examples}

The restriction that we mentioned regarding construction of quaternionic manifolds may pose a difficulty in having explicit examples. However string theory gives us a very simple way to construct quaternionic manifolds that are consistent with the $c$-map:

- Construct a vector multiplet lagrangian in four dimensions. The multiplet is $\left(A_{\mu}, 2 \phi, 2 \psi\right)$ with the real-scalars forming a Kähler target space. Such a lagrangian coupled to gravity is well known [16].

- Dimensionally reduce this lagrangian to three spacetime dimensions. The vector multiplet will give us $\left(A_{\mu}, 3 \phi, 2 \psi\right)$ in three dimensions.

- Dualise the vector to another scalar $\varphi$ via $d \varphi=* d A$ to convert the vector multiplet to a hypermultiplet $(4 \phi, 2 \psi)$. The metric on the moduli space of these scalars is exactly quaternionic [6].

\footnotetext{
4 Or sometime as the $s$-map [5].

5 We thank Ulrich Theis for pointing this out to us.
} 
- The quaternionic metric is also consistent with the $c$-map because we derived this from the vector multiplet with a Kähler target. Thus the quaternionic manifold will have a submanifold that is Kähler, as one would have expected [6].

In fact the above set of steps can be put into a more concrete setting. Consider a simple $\mathcal{N}=2$ lagrangian with complex scalars coupled to one forms and gravity. A typical set up is

$$
S_{4}=\int d^{4} x \sqrt{g}\left[R+G_{a \bar{b}} \partial_{\mu} \phi^{a} \partial^{\mu} \bar{\phi}^{b}+c_{i j} F^{i} \wedge * F^{j}\right]+d_{i j} F^{i} \wedge F^{j}
$$

where $G_{a \bar{b}}$ is the metric on the moduli space - which will be a Kähler metric as we discussed above - and $c_{i j}$ and $d_{i j}$ are some coefficients which are functions of the moduli $\phi^{a}$. The subscript $i, j$ signify the number of vector multiplets that we couple to gravity.

In this form the lagrangian (2.12) is almost like a D3-brane action coupled to gravity. However the resulting configuration should not be viewed as a D3 located at a point on a Calabi-Yau because the supersymmetry will not be $\mathcal{N}=2$ and the dimension of the Kähler moduli space will be fixed. Furthermore the instanton coefficient $d_{i j}$ is not quite related to the ten-dimensional axion. We will however relate a slight variant of this configuration to a D3 brane metric soon.

After a dimensional reduction and subsequent duality, we will get a three dimensional action for the hypermultiplets. This is given by:

$$
S_{3}=\int d^{3} x \sqrt{g}\left[R+G_{a \bar{b}} \partial_{\mu} \phi^{a} \partial^{\mu} \bar{\phi}^{b}+\mathcal{G}_{c \bar{d}} \mathcal{D}_{\mu} \varphi^{c} \mathcal{D}^{\mu} \bar{\varphi}^{d}\right]
$$

where $(\phi, \varphi, \bar{\phi}, \bar{\varphi})$ form the coordinates of a quaternionic space with a metric $\mathcal{G}_{c \bar{d}}$ spanning the submanifold specified by the coordinate $\varphi^{c}$. The covariant derivatives $\mathcal{D}_{\mu} \varphi^{c}$ are with respect to some connection. This structure of the moduli space can be easily connected to the ones studied by [20], [17].

We can try to make this a bit more precise using the previous form of our action (2.6). Let us consider the following choice of the quaternion:

$$
q=\left(\begin{array}{cc}
0 & B \\
C & 0
\end{array}\right)
$$

where both $B$ and $C$ are complex numbers (not necessarily independent). The scalar target space parametrised by the quaternion then will have the following structure:

$$
\mathcal{L}=\frac{\left|\partial_{\mu} C\right|^{2}+\left|\partial_{\mu} B\right|^{2}}{|C|^{2}+|B|^{2}}-\frac{\left|C \partial_{\mu} C^{*}+B \partial_{\mu} B^{*}\right|^{2}}{\left(|C|^{2}+|B|^{2}\right)^{2}}
$$


where we have suppressed the gravity part. Consider now the scenario where $B$ and $C$ appearing above are complex numbers, but are not independent. They are related by

$$
B=-C^{*}
$$

as is clear from the quaternionic structure of the $q$ coordinate. Such a choice of $B, C$ would imply that the lagrangian (2.15) can be recast as

$$
\mathcal{L}=\frac{2\left|\partial_{\mu} C\right|^{2}}{\mathcal{S}+\mathcal{S}^{*}}-\frac{\left|\partial_{\mu} \mathcal{S}\right|^{2}}{\left(\mathcal{S}+\mathcal{S}^{*}\right)^{2}}
$$

where, in our notation, $\mathcal{S}$ is not quite an independent variable as it stands. It is given by

$$
\mathcal{S}=|C|^{2}
$$

The reason for writing (2.17) in the present form is to allude to the subsequent structure that we will be inferring from string theory.

The string theory examples that have been studied earlier are all non-compact symmetric spaces with negative curvatures. In fact string theory tells us precisely how $S$ defined above (2.18) should be modified so as not to change the underlying quaternionic structure. The resulting metric will be consistent with the target space metric of a tensor multiplet $\left(B_{\mu \nu}, 3 \phi, 2 \psi\right)$ when dualised to a hypermultiplet in four dimensions. Although this is no way the most generic method to derive the metric, it does help us to see the subsequent structure. In type IIA this is therefore a compactification on a Calabi-Yau three-fold that has no complex structure deformations (more on this later). Furthermore since dilaton sits precisely in such a multiplet, quantum corrections are expected to affect the target space metric. After the dust settles, the final answer is a slight modification of our simple calculation above. The quantity $S$ changes from (2.18) to

$$
\mathcal{S}=|C|^{2}+e^{-2 \phi}+i \varphi
$$

where $\phi$ is the dilaton sitting in the tensor multiplet, $\varphi$ is the corresponding axion (dualised from the $B_{\mu \nu}$ field in four dimensions) and $C, C^{*}$ are the other two scalars in the tensor multiplet. These are the two scalars that come from type IIA three form in ten dimensions. Similarly the Kähler potential is changed to

$$
\mathcal{K}=-\ln \left(\mathcal{S}+\mathcal{S}^{*}-2|C|^{2}+\text { quantum corrections }\right)
$$


which implies that the resulting manifold is also Kähler (see [17 for some details). Without quantum corrections the tree level moduli space for the universal hypermultiplet is given by

$$
\mathcal{M}_{\mathrm{H}}=\frac{S U(1,2)}{U(2)}
$$

which is the non-compact analogue of $\mathbf{G r}_{2}\left(\mathbf{C}^{3}\right)$ because of the negative curvature. Under tree level quantum corrections the Kähler structure of the moduli space is broken [21]. Further corrections to the moduli space come from the two- and five-brane instantons. These and others have been addressed in [18], [19], [22] as we discussed briefly before, although a full treatment is far from complete.

Let us consider another example. This time we compactify type IIA theory on a Calabi-Yau threefold with no complex structure deformations (i.e $h_{21}=0$ ). Thus in four dimension we will have the following multiplet structure:

$$
\left(g_{\mu \nu}, A_{\mu}\right) \oplus h_{11}\left(A_{\mu}, 2 \phi\right) \oplus\left(B_{\mu \nu}, 3 \phi\right)
$$

which is a slight modification of (2.9). As we can see, the universal hypermultiplet is always there. The moduli space therefore is from the vector multiplet Kähler space as well as the universal hypermultiplet, as is given by

$$
\mathcal{M}=\mathcal{G}_{\text {Kahler }}^{h_{11}} \otimes \frac{S U(2,1)}{U(2)}
$$

where $\mathcal{G}$ is the Kähler manifold of dimension $h_{11}$. Observe also the fact that there are $\left(1+h_{11}\right)$ vectors in this setup (extra one coming from the gravi-photon).

Compactifying type IIB theory on the same Calabi-Yau gives us $\left(1+h_{11}\right)$ hypermultiplets coupled to gravity (and graviphoton) and no vector multiplets. The quaternionic manifold that we get here can in fact be derived from the moduli space (2.23) via the $c$-map. This is given by

$$
\mathcal{G}_{\text {quaternion }}^{4\left(h_{21}+1\right)}
$$

from where we can easily see that the quaternionic space $\frac{S U(2,1)}{U(2)}$ forms a sub-manifold of the final irreducible quaternionic space $\mathcal{G}^{4\left(h_{21}+1\right)}$. This is the essence of the $c$-map in the presence of the universal hypermultiplet.

In the following section we will address the question of classifying quaternionic manifolds using constrained instantons and Seiberg-Witten curves, and discuss the emergence of the so-called magic square. 


\section{On the classification of quaternionic manifolds: standard cases}

As discussed in earlier sections, the classification of quaternionic manifolds have been started in [2], [3], and completed finally in [23]. Many of the cases that we studied so far (or have been addressed in the literature) can be seen to follow from the above framework. For our case we will try to understand the classification of the compact symmetric quaternionic Kähler manifolds using a different technique. Some aspects of this have been addressed earlier in [24].

\section{1. $S p(n+1)$ quaternionic space}

Our first starting point will be the simplest case of $S p(n+1)$ quaternionic space 6 . As we will discuss below, the quaternionic space associated with $S p(n+1)$ group is special in the whole classification of quaternionic spaces. The key point that we will follow to classify these spaces is this: we look for gauge theories with certain global symmetries $\mathcal{G}$ (here, for this case, it is $S p(n+1)$ ) and find semi-local instanton configurations. The low momentum dynamics of these theories (by low momenta we mean momenta lower than the masses of the Higgs and the masses of the photons) can be shown to be sigma models with quaternionic target spaces. Such an approach was first discussed in [25] (see also [26] for sigma models on Kähler target spaces) and later elaborated in [24]. Here we will try to complete the analysis by detailing the corresponding gauge theory constructions.

The gauge theory that we are looking for is an $S p(1) \equiv S U(2)$ gauge theory with a global symmetry $\mathcal{G}$. Clearly this theory resembles closely to a sector of the corresponding Seiberg-Witten theory with global symmetries [8]. To make this precise, let us write the action for our theory. This is given by the following generic form 25:

$$
S=\int d^{4} x\left[\frac{1}{4} \operatorname{tr}_{\mathrm{SU}(2)}\left(F_{\mu \nu} F^{\mu \nu}\right)+\operatorname{tr}\left(D_{\mu} q^{\dagger} \cdot D^{\mu} q\right)+V\left(\operatorname{tr}\left(q^{\dagger} \cdot q\right)\right)+\text { fermions }\right]
$$

where $q$ is a generic quaternion as described in the previous section, and the trace is over the global symmetryt. Obviously, as mentioned above, this is not quite a Seiberg-Witten

6 A point about notation: we will be considering $S p(n)$ groups instead of $S p(2 n)$ groups used sometime in the literature. In our notation therefore $S p(n)$ group is just the quaternionic unitary group $U(n, \mathbf{H})$. Its a real, compact and simply connected Lie group of dimension $n(2 n+1)$. In particular $S p(1) \equiv S U(2)$ and we will not distinguish between them in this paper.

7 Note that $q$ will transform as a fundamental of both the global $\mathcal{G}$ and the local $S U(2)$ groups for all choices of $\mathcal{G}$ considered henceforth unless mentioned otherwise. 
theory as it stands. However once we write the quaternions in terms of complex fields (we show an example below), the action will resemble a part of the standard $\mathcal{N}=2$ action with a potential $V$ (a simple case is the one worked out in [27] for an $S p(1)_{g} \times S p(1)_{l}$ case). In this sense, we can use the Seiberg-Witten curves to determine the global properties of this model. A recent example of semilocal defects like strings in Seiberg-Witten theory is [28]. Our goal is to study instantons in the model (3.1) i.e a sector of, and not quite the actual, Seiberg-Witten theory. In fact the analysis of instantons in this theory can be done in two different ways, both leading to the same result. The first way is to observe that a theory like (3.1) will not allow any non-trivial instantons if

$$
\pi_{3}\left(\frac{\mathcal{G}}{\mathcal{H}}\right)=1
$$

where $\mathcal{H}$ is the unbroken subgroup. However instantons are possible when a subgroup of $\mathcal{G}$ is gauged 8 . Let us call the ungauged subgroup of $\mathcal{G}$ to be $\mathcal{G}_{g} \equiv \mathcal{H}$. Then the vacuum manifold $\mathcal{M}_{1}$ of this theory is rather simple. It is given by:

$$
\mathcal{M}_{1}=\frac{\mathcal{G}}{\mathcal{G}_{g}}=\frac{S p(n+1)}{S p(n)} \approx \mathbf{S}^{4 n+3}
$$

where, as should be clear from the above analysis, $\mathcal{G}_{g}=S p(n)$ and we are taking the following breaking pattern:

$$
\frac{S p(n+1)_{g} \times S p(1)_{l}}{\mathbf{Z}_{2}} \stackrel{\Phi}{\longrightarrow} \frac{S p(n)_{g} \times S p(1)_{g}}{\mathbf{Z}_{2}}
$$

with $\Phi$ being the Higgs field. The Higgs field is to be considered as a quaternion and not a complex number, although we could consider this also to be a complex matrix. The quaternion that could be used to represent the Higgs field is already pointed out above in (2.5). Thus

$$
\Phi \equiv\left(q_{a}\right)=\left(\begin{array}{cc}
-\phi_{a}^{1} \phi_{a}^{2 *} & \left|\phi_{a}^{1}\right|^{2} \\
-\left|\phi_{a}^{2}\right|^{2} & \phi_{a}^{2} \phi_{a}^{1 *}
\end{array}\right)
$$

is a good representation of the Higgs field in terms of the quaternions $\left(q_{a}\right)=\left(q_{1}, q_{2}, \ldots, q_{n}\right)$ or in terms of $\phi_{a}$. As pointed out in [25], [10] (and references therein) this is equivalent to a model with $n+1$ copies of the electroweak scalar sector with an $S p(n+1)$ global symmetry in the $\theta_{W}=0$ limit.

8 These are the constrained instantons [9] as we will explain below. 
The second way is to view (3.1), when written in terms of complex coordinates and incorporating other terms, as describing the Higgs branch of Seiberg-Witten theory. Then the semilocal instantons can be related to the small instantons described by Witten [29] and Ganor-Hanany [30] and the vacuum manifold $\mathcal{M}_{1}$ becomes the moduli space of oneinstanton. These instantons are described by embedding $S U(2)$ groups inside the global groups, and therefore the different $S U(2)$ orientations describe the moduli space of the theory 9 . These $S U(2)$ orientations form an $S^{3}$ and the moduli associated with the sizes of these instantons form the radii of the three cycles. In this language these three cycles will be fibered over quaternionic Kähler spaces. Such an approach has been used to study quaternionic Kähler manifolds associated with $A_{n}, B_{n}, C_{n}$ and $D_{n}$ groups [34]. The moduli space then is a 3-Sasakian spaces that are $S p(1)$ fibrations over quaternionic Kähler spaces 355 and is given by:

$$
\mathcal{M}_{k}=\mathbf{R}^{4} \times \mathbf{R}^{+} \times\left[S p(1) \otimes_{f} \mathbf{Q}_{k}\right]
$$

where $\mathbf{R}^{4}$ denotes the four-translation moduli, $\mathbf{R}^{+}$denotes the size moduli, the subscript $k$ denotes $k$-instantons, $\mathbf{Q}_{k}$ denotes the quaternionic space associated with $k$-instantons and the subscript $f$ denotes non-trivial fibration. In the following we will give a concrete example of such fibration using mostly the first technique (although in many cases we will alternate between the two techniques10). This will prove convenient for theories that

9 Observe that, if we view the Seiberg-Witten theory to be generated by D3/D7 system a la [31], then the gauge instantons are $D(-1)$ branes inside $D 3$ branes, whereas the small instantons are the bound states of $D 3$ branes with the $D 7$ branes [32]. If we T-dualise the system then we will have a configuration of $D 1, D 5$ and $D 9$ branes. The moduli space of the small instantons on $D 9$ branes i.e $D 5$ branes in $D 9$ branes is given via ADHM data by a special hyper-Kähler manifold, or a quaternionic Kähler manifold when coupled to supergravity [29]. On the other hand the moduli space of $D 1$ branes is given by a sigma model with ADHM target space [32], [33. Thus both the pictures describe the same physics.

10 There is also a third way of studying the moduli spaces of these instantons that is slightly different from the above two approaches (although more related to the second one). This has to do with the fact that $\mathcal{N}=2$ supersymmetric gauge theories also have hypermultiplets in the adjoint representations of the gauge groups. Observe that the hypermultiplets that we considered for the above two cases are all in the fundamental representations of the gauge groups. Combining these adjoint hypermultiplets with the $\mathcal{N}=2$ vector multiplets will give us the spectrum of $\mathcal{N}=4$ gauge theories. In these theories moduli spaces of instantons will be exactly the same as for the fundamental hypermultiplets if we exchange the global symmetries with gauge symmetries. Thus $\mathcal{N}=4$ theories with exceptional gauge symmetries will have the same moduli spaces of instantons 
may not have a good Lagrangian description (and therefore no well defined Higgs branch) but more importantly the technique of semilocal defects is ideally suited to study other manifolds in the magic square as we will discuss soon. Below we tabulate the precise connection between our semilocal theory, and the full Seiberg-Witten theory:

\begin{tabular}{|c|c|}
\hline Semilocal Theory & Seiberg-Witten Theory \\
\hline Semilocal instantons & Small instantons \\
\hline $\mathrm{G} / \mathrm{H}=$ Vacuum manifold & $\begin{array}{l}\text { Higgs branch = Instanton moduli space } \\
=\text { Special Hyper-Kahler manifold }\end{array}$ \\
\hline $\begin{array}{l}\text { Gauging an } \mathrm{SU}(2) \text { subgroup } \\
\text { of the global group }\end{array}$ & \multirow{2}{*}{$\begin{array}{l}\text { Embedding SU(2) instanton in the } \\
\text { global group = Orienting SU(2) group } \\
\text { in a global group }\end{array}$} \\
\hline$\Pi_{3}(\mathrm{G} / \mathrm{H})=1, \Pi_{3}(\mathrm{SU}(2))=\mathrm{Z}$ & \\
\hline $\mathrm{H}=$ Unbroken subgroup & $\mathrm{H}=$ Stability group of the instanton \\
\hline Quaternions & $\begin{array}{c}\mathrm{N}=1 \text { chiral multiplets or } \mathrm{N}=2 \\
\text { hypermultiplets }\end{array}$ \\
\hline SU(2) gauge group & Microscopic SU(2) group \\
\hline Quaternionic Kahler manifolds & Quaternionic Kahler manifolds \\
\hline Semilocal strings & Semilocal strings \\
\hline Mass term in the potential & $\begin{array}{l}\text { Massive hypers, mixed Coulomb-Higgs } \\
\text { branch }\end{array}$ \\
\hline
\end{tabular}

From above table it should be clear that although our theory (3.1) is a small sub-sector of the original Seiberg-Witten theory, it has all the necessary ingredients to understand the detailed aspects of magic square as we will demonstrate soon. The complicacies of the full Seiberg-Witten theory, for example the existence of Coulomb branch or mixed CoulombHiggs branch, do not effect the analysis that we are going to perform therefore we will continue with our simpler version (3.1) 1 . However we will try to demonstrate, whenever possible, how to analyse the system from the full Seiberg-Witten theory.

as we study here. Such an approach has been discussed by Stefan Vandoren in the last reference of [34]. For most of our analysis in this paper we will not consider the adjoint hypermultiplets as we want to analyse $\mathcal{N}=2$ gauge theories only.

11 In fact our model (3.1) doesn't have a Coulomb branch. So in the corresponding SeibergWitten theory this is the pure Higgs branch. 
Thus having laid down the possible criteria to construct explicit $S p(n+1)$ quaternionic manifolds, there are a few important points to analyse now:

- We have to verify whether it is possible to construct a Seiberg-Witten like theory with $S p(n+1)$ global symmetry. This would be confirmed by the existence of the corresponding Seiberg-Witten curve for the system. We expect, on generic ground, a curve of the form:

$$
y^{2}-x^{3}-a_{2} x^{2} k(z)+a_{1} x y l(z)+a_{3} y h(z)-a_{4} x f(z)-a_{6} g(z)=0
$$

with $a_{i}$ being constants and $k(z), l(z), h(z), f(z)$ and $g(z)$ are polynomials in $z$. The coordinate $z$ specifies the complex plane in the corresponding Seiberg-Witten theory. The above equation with the right choice of $k, l, h, f$ and $g$ takes the following Weierstrass form that reflects an $S p(n+1)$ global symmetry:

$$
y= \pm \sqrt{x^{3}+x z^{n+1}+\frac{5}{4} z^{2 n+2}}-\frac{z^{n+1}}{2}
$$

Using this one can check that the curve 12 has the right singularity structure to allow an $S p(n+1)$ global symmetry. A similar curve should then describe the global properties of our model.

- The next step to verify would be the existence of instantons in this model. Clearly existence of the corresponding curve (3.8) means that we have summed all the instanton contributions to get the required Seiberg-Witten curve. However it is instructive to actually construct these instantons. Out of the various different possibilities of instanton configurations in our system (because of the matter representations) we will henceforth only concentrate on the so-called semilocal instantons unless mentioned otherwise. These are the small instantons in the Higgs branch of the full theory. Their construction is subtle because of two reasons. Firstly the vacuum manifold being $S^{4 n+3}$ would imply

$$
\pi_{3}\left(\mathcal{M}_{1}\right)=1
$$

12 Observe that this is only a genus one curve. For higher local gauge symmetry, for example $S U(N)$ with $N>2$, we will have a genus $N-1$ curve. In this paper we will look mostly at the sector of the theory that is given by a genus one (i.e $N=2$ ) curve although in the last part of section 4 we will give some examples of higher genus curves. Generic case of an $S U(N)$ gauge theory broken to $S U(2) \times G_{\text {local }}$ gauge theory will be studied in the sequel to this paper. 
so would disallow instantons. The only allowed instanton configurations therefore would be the semilocal instantons by gauging an $S p(1)$ part of the global symmetry 13 . We may then expect that the low momentum dynamics of the theory should be a sigma-model on a certain quaternionic space, or alternatively the moduli space of the Higgs branch instantons should be given by the quaternionic space. The structure of the corresponding quaternionic space can be determined from the following gauge field configuration:

$$
A_{\mu} \equiv A_{\mu}^{a} \sigma^{a}=\frac{1}{2 g_{\mathrm{YM}}^{2}} \cdot \frac{q^{\dagger} \cdot \partial_{\mu} q-q \cdot \partial_{\mu} q^{\dagger}}{\operatorname{tr}\left(q^{\dagger} \cdot q\right)}
$$

where the sum over repeated indices are implied via the dot product and $\sigma^{a}$ are the Pauli matrices. Now due to the existence of F- and D-terms the low energy effective action will be a quaternionic manifold $\mathbf{H P}^{n}$ as shown in (2.6) when (3.10) is plugged in the action (3.1). The semilocal instantons in this model have the following structure (see also [25]):

$$
\pi_{3}\left(S^{3}\right)=\mathbf{Z}, \quad S^{4 n+3} \stackrel{S^{3}}{\longrightarrow} \mathbf{H P}^{n}
$$

provided certain subtleties are considered. This is the second reason. The subtlety has to do with the presence of $V\left(\operatorname{tr}\left(q^{\dagger} \cdot q\right)\right)$ term in the action (3.1), namely, due to Derrick's theorem once the scale invariance is broken by a mass term in the potential, the instantons all squeeze to zero size. So the semilocal instantons that we are alluding to should exactly be the constrained instantons of Affleck [9]. These constrained instantons resemble the standard instanton at short distances only but decay exponentially at the IR [9] (see also [36]). In the notation of [27], when

$$
S=\zeta_{ \pm}=\zeta_{3}=0
$$

where $\zeta$ are the FI terms, then the instanton allowed are the standard instantons. For the case when the FI terms are non-zero, to construct constrained instantons all we require is the maximal subalgebra of the extended Dynkin diagram of $S p(n+1)$ :

13 One might be wondering about the connection between the curve (3.8) and the contributions from the semilocal instantons. As is well known all possible instantons should contribute to the path integral to determine the full curve of the theory [33]. The curve (3.8) is the minimal curve with $S p(n+1)$ global symmetry so will have contributions from the semilocal instantons (which are of course the small instantons in the Higgs branch). The situation gets tricky when the global symmetry becomes very large (for example $E_{n}$ as we will encounter later). In those situations how exactly all the instantons contribute to give us the full curve will be described elsewhere. 


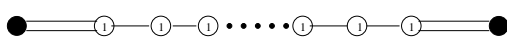

should be expressible as a product of two subalgebras. This fixes the maximal subalgebra for our case to be $s p(n) \oplus s p(1)$. The constrained instantons are exactly of the gauged $S p(1) \equiv S U(2)$ group. The simplest non-trivial example of such an instanton is for the global group $S p(2)$. The quaternionic space associated with this global group is a four sphere $S^{4}$ because:

$$
\frac{S p(2)}{S p(1) \times S p(1)}=S^{4} \equiv \mathbf{H P}^{1}
$$

and therefore the constrained instantons are non-trivially fibered over the four sphere (this has also been noticed for a non-stringy example in [25]). For our case when $\zeta_{3} \neq 0$ and

all other FI terms vanishing in $V\left(\operatorname{tr}\left(q^{\dagger} \cdot q\right)\right)$ of (3.1), the constrained instanton can be explicitly worked out to be of the following form:

$$
A_{\mu}=\frac{2 \rho^{2} \sigma^{a} \eta_{\mu \nu}^{a} x_{\nu}}{x^{2}\left(x^{2}+\rho^{2}\right)}-\frac{\zeta_{3} g_{\mathrm{YM}}^{2}}{2} \cdot \frac{\sigma^{a} \eta_{\mu \nu}^{a} x_{\nu}}{x^{2}}+\ldots
$$

where $\rho^{2}$ is the typical size of the instanton in the scale invariant limit (which is of course the $\zeta_{3}=0$ limit). Observe that we need to also switch on non-zero expectation values for the quaternions. It can be easily shown that the background values of the quaternions are always proportional to the FI term $\zeta_{3}$ so that in the scale invariant limit their expectation values have to vanish to allow the standard instantons to exist. In the figure below:

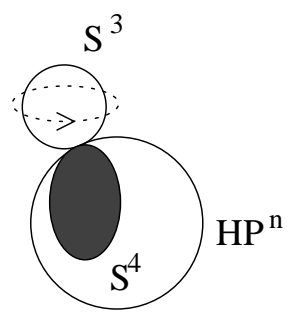

a typical constrained instanton is shown. We see that the instanton is non-trivially fibered over the quaternionic base $\mathbf{H} \mathbf{P}^{n}$ and wraps the three sphere $S^{3}$ once at infinity. Over the rest of the space it completes a non-trivial four sphere $S^{4}$ in the quaternionic space. This also means that for $\mathbf{H P}^{1}$ we will have a controlled theoretical way to study the instanton. This is in fact further facilated by the following group theory identities:

$$
\mathbf{H P}^{1}=S^{4}=\frac{S O(5)}{S O(4)}
$$


which means that this special case could even be studied using real fields. This is indeed the case, and has been attempted in [25].

The above set of procedures was to construct a configuration of the simplest quaternionic space $\mathbf{H P}^{n}$ using constrained instantons. The relevant non-compact extension of the above space is the quaternionic space

$$
\frac{S p(n, 1)}{S p(n) \times S p(1)}
$$

which is more useful to study the moduli spaces in type II theories. Now recall that there is a natural one-to-one correspondence between quaternionic normal Lie algebras and quaternionic simply connected normal homogeneous spaces. In fact any normal quaternionic algebra should contain a one-dimensional quaternionic subalgebra called the canonical quaternionic subalgebra. The manifold that we studied above (3.16) correspond to the following totally geodesic subalgebra:

$$
C_{1}^{1} \equiv \frac{S p(1,1)}{S p(1) \times S p(1)}
$$

In fact (3.16) is the unique quaternionic algebra whose canonical subalgebra is isomorphic to $C_{1}^{1}$ [3]. However there is no Kähler space associated with (3.16) because there is no c-map. So (3.16) cannot appear as low energy lagrangian in type II theories. Thus our construction of the corresponding compact $\mathbf{H P}^{n}$ gives the only legitimate way to study this manifold in string theory. Below we will show that all the compact versions of the symmetric quaternionic spaces can be studied using the technique of constrained instantons. In fact we will show how the magic square appears in this analysis. But first, lets go to the next non-trivial example related to the $G_{2}$ quaternionic space.

\section{2. $G_{2}$ quaternionic space}

The technique that we developed in the previous subsection is universal. We will use

the same procedure of constrained instantons to construct quaternionic manifolds for the $G_{2}$ cases also. However instead of repeating the same constructions once again, we will give a concrete mathematical way to build the quotient space:

$$
\frac{G_{2}}{S p(1) \times S p(1)}
$$


so that combining this procedure and the steps elucidated in the previous subsection we will be able to classify the magic square cases in the next section.

Before going into the details of the specific construction of (3.18) we would like to make the following comments. The quotient structure of (3.18) should be obvious from the previous analysis, namely, the maximal subalgebra of $G_{2}$ without an $U(1)$ factor from the extended Dynkin diagram:

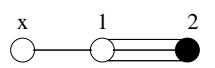

is $s o(4) \equiv s u(2) \oplus s u(2)$. As this is already expressed in terms of two product group (with an $s u(2)$ factor) we needn't go any further. In fact the 7 of $G_{2}$ then decomposes as 14 :

$$
7 \rightarrow(2,2)+(1,3)
$$

under $S U(2) \times S U(2)$, where once we give a $\operatorname{VEV}$ to $(\mathbf{2}, \mathbf{2})$ one of the global $S U(2)$ (which is broken) mixes with the broken local $S U(2)$ to give us a diagonal unbroken $S U(2)$. The quotient space is then clearly (3.18). What remains to study however is the precise embedding of the $S U(2)$ groups inside a $G_{2}$. This will be addressed below.

The next issue is the existence of the corresponding Seiberg-Witten curve for a global $G_{2}$ group. We have already laid down the possible curve for any global group $\mathcal{G}$ in (3.7). For $\mathcal{G}=G_{2}$ we can choose certain specific functional form for $k, l, h, f$ and $g$ in (3.7) to give us the following curve:

$$
\begin{aligned}
\left(y+\frac{12 a_{1} z x-4 a_{1} a_{2} z^{2}-4 a_{1}^{2} z^{2}+12 a_{3} z^{2}}{24}\right)^{2}=x^{3}-\frac{x}{48}\left[a_{1}^{4} z^{4}+8\left(a_{1}^{2} a_{2}-3 a_{1} a_{3}-6 a_{4}\right) z^{3}+\right. \\
\left.+16 a_{2}^{2} z^{2}\right]+\frac{1}{864}\left[a_{1}^{8} z^{8}+12\left(a_{1}^{4} a_{2}-3 a_{1}^{3} a_{3}\right) z^{5}+\left(48 a_{1}^{2} a_{2}^{2}+216 a_{3}^{2}-72 a_{1}^{2} a_{4}-\right.\right. \\
\left.\left.-144 a_{1} a_{2} a_{3}\right) z^{4}+\left(64 a_{2}^{3}-288 a_{2} a_{4}+864 a_{6}\right) z^{3}\right]
\end{aligned}
$$

where $a_{i}$ are some constants. The precise mapping of this curve to the $G_{2}$ Casimirs can be worked out but we will not do so here as our emphasis is more on the magic square. One can check that the discriminant is

$$
\Delta \sim z^{6}+\mathcal{O}\left(z^{8}\right)
$$

14 We thank Tom Kephart for discussions on this point. 
and therefore reflects a global $G_{2}$ symmetry near the point $z=0$. To see the full global symmetry for other cases one has to generalise the above curve (3.20) further. Examples of these will be discussed in the next section.

Another point is the existence of third homotopy groups for various coset spaces. For a global group $\mathcal{G}$ broken to a subgroup $\mathcal{H} \times S U(2)$ our first criteria would be to ask the value of the third homotopy from the exact sequence

$$
\longrightarrow \pi_{3}(\mathcal{H}) \longrightarrow \pi_{3}(\mathcal{G}) \longrightarrow \pi_{3}(\mathcal{G} / \mathcal{H}) \longrightarrow 0
$$

where both $\mathcal{G}, \mathcal{H}$ are Lie groups 15 . For simple cases dealing with non-exceptional groups this is easy and well known. The interesting question comes when $\mathcal{G}$ is an exceptional group or when both $\mathcal{G}$ and $\mathcal{H}$ are exceptional groups. Three rules have been developed to address these questions [37]:

- When both $\mathcal{G}$ and $\mathcal{H}$ are simple, i.e when both $\mathcal{G}$ and $\mathcal{H}$ do not have invariant Lie subgroups, then

$$
\pi_{3}(\mathcal{G} / \mathcal{H})=\mathbf{Z}_{M}, \quad M \equiv \frac{l}{L}
$$

where $L$ is a non-negative integer called the index of a representation $\mathcal{D}_{\mathcal{G}}$ for the group $\mathrm{G}$. Similarly $l$ is the index for the corresponding representation $\mathcal{D}_{\mathcal{H}}$ for the group $\mathcal{H}$. These indexes are tabulated in details for many representations in 38 . The idea is to look for a particular representation (say vector or tensor) for the group $\mathcal{G}$ and then look for the same representation for the group $\mathcal{H}$. The ratio of the corresponding indexes will give us the value for $\pi_{3}(\mathcal{G} / \mathcal{H})$. It is interesting to note that as long as we choose the same representations for both $\mathcal{G}$ and $\mathcal{H}$ the ratio $l / L$ will always be the same.

- If $\mathcal{G}$ is simple but $\mathcal{H}$ is of the form of $\mathcal{H}_{1} \otimes \mathcal{H}_{2} \otimes \ldots \mathcal{H}_{n}$ with $\mathcal{H}_{i}$ simple, then

$$
\pi_{3}(\mathcal{G} / \mathcal{H})=\mathbf{Z} \bmod \text { every } \frac{l_{i}}{L}
$$

15 This is crucial because, as mentioned earlier, our theory is only a sector of a bigger theory. Consistency requires that we evaluate the third homotopy of $\frac{\mathcal{G}}{\mathcal{H}}$ to study the instantons. On the other hand, in the full Seiberg-Witten theory, the instantons are in the Higgs branch and so we would only require to evaluate the third homotopy of the global group $\mathcal{G}$. For more details see the table of comparison given earlier.

16 These indexes are representated as $\frac{I(2)}{\text { rank }}$ in [38]. 
where $\left(l_{1}, l_{2}, \ldots, l_{i}\right)$ are the collection of n-tuples. In fact $\mathcal{H}$ can have an additional abelian subgroup without changing the result. Furthermore modding by a discrete subgroup also doesn't change the result.

- When both $\mathcal{G}$ and $\mathcal{H}$ are not simple and $\mathcal{G}$ is of the form $\mathcal{G}_{1} \otimes \mathcal{G}_{2} \otimes \ldots \mathcal{G}_{n}$ where $\mathcal{G}_{i}$ are simple 17 , then $\pi_{3}(\mathcal{G} / \mathcal{H})$ consists of $n$-tuples of the form

$$
\left(\sigma_{1}, \sigma_{2}, \ldots, \sigma_{n}\right) \bmod \text { every }\left[\frac{l_{i}^{(1)}}{L_{1}}, \frac{l_{i}^{(2)}}{L_{2}}, \ldots, \frac{l_{i}^{(n)}}{L_{n}}\right]
$$

where $\left(l_{i}^{(1)}, l_{i}^{(2)}, l_{i}^{(2)}, \ldots, l_{i}^{(n)}\right)$ are the $n$-tuples associated with the simple groups $\mathcal{H}_{i}^{(1)}, \mathcal{H}_{i}^{(2)}, \ldots$ etc., where the Lie algebras $g, g_{i}, h_{i}$ associated with the Lie groups $\mathcal{G}, \mathcal{G}_{i}, \mathcal{H}_{i}$ respectively have the decomposition $h_{i}=\oplus_{j} h_{i}^{(j)}$ with the condition $h_{i}^{(j)} \subseteq g_{j}$. The Lie algebras $h_{i}^{(j)}$ are either isomorphic to $h_{i}$ or $\{0\}$. For more details the readers may want to refer to [37], and [38].

Therefore the upshot of all these discussions is that the third homotopy groups for coset spaces can either be 1 or $\mathbf{Z}_{p}$. For exceptional groups the third homotopy groups are all Z. In fact generically $\left.\pi_{3}(S U(n))\right|_{n \geq 2}=\mathbf{Z}$. Similarly $\left.\pi_{3}(S O(n))\right|_{n \geq 3, n \neq 4}=\mathbf{Z}$ and $\pi_{3}(S O(4))=\pi_{3}(S U(2) \times S U(2))=\mathbf{Z} \oplus \mathbf{Z}$. This would mean that $\pi_{3}\left(G_{2} / S U(2)\right)=1$ i.e the third homotopy group is trivial 18 , although this doesn't mean much because with $G_{2}$ global symmetry a lagrangian description of the system like (3.1) discussed previously is not possible 19 . Therefore to study the constrained instantons in the system we gauge the

17 Additionally allowing abelian groups as well as discrete moddings.

18 It turns out there are other possible embeddings of an $S U(2)$ group in $G_{2}$, namely that the $\mathbf{7}$ of $G_{2}$ goes to $\mathbf{3}+\mathbf{2}+\mathbf{2}$ of $S U(2)$ or the $\mathbf{7}$ of $G_{2}$ goes to $\mathbf{7}$ of $S U(2)$. For these two cases $\pi_{3}\left(G_{2} / S U(2)\right)=\mathbf{Z}_{3}$ or $\mathbf{Z}_{28}$ respectively. We thank V.P. Nair for pointing this out to us.

19 It is an issue - and we will discuss this again later - for all theories with exceptional global symmetries. One can see this from the D3/D7 brane construction of these theories. The fundamental hypermultiplets appear from the strings connecting the D3 branes with the D7 branes. The gauge symmetries of the seven brane theories appear as global symmetries of the underlying D3 brane theories. For classical Lie groups as gauge or global symmetries, the seven branes are all D7-branes. However when we have exceptional Lie groups, not all seven branes are D7 branes. Some of them are $S L(2, \mathbf{Z})$ transform of the D7-branes. Because of that strings connecting the D3 and the seven branes may take non-trivial paths in the $u$-planes of corresponding Seiberg-Witten theories [39]. For such strings simple Born-Infeld action may not be easy to write down. Nevertheless such theories exist as can be easily shown from the corresponding F-theory, or the Seiberg-Witten curves. Since the curves are constructed by summing up all the instantons, 
$S U(2)$ subgroup of the maximal $S U(2) \times S U(2)$ group, or alternatively - viewing this in the Higgs branch - we study the orientations of $S U(2)$ inside $G_{2}$. Thus effectively we are studying $S U(2)$ constrained instantons in a theory with the maximal group. These instantons are non-trivially fibered over the base (3.18).

As we discussed for the $S p(n+1)$ case in the previous section we can now describe a possible quaternionic geometry associated with the constrained instantons. In fact, as before, we need the sigma model on the non-compact version of the geometry namely, on

$$
\frac{G_{2(+2)}}{S U(2) \times S U(2)}
$$

To determine this we can use the trick of the $c$-map, that uses the metric of the Kähler manifold to determine the quaternionic manifold. The Kähler manifold and the associated $F$ function in question are [6], [5], [16]:

$$
\mathcal{M}_{\text {Kahler }}=\frac{S U(1,1)}{U(1)}, \quad F\left(X^{I}\right)=\frac{i\left(X^{2}\right)^{3}}{X^{1}}
$$

where $X^{I}, I=1,2, \ldots, n+1$ are the scalar fields corresponding to certain other $\mathcal{N}=2$ vector multiplets (including the gravi-photon) and we have introduced the $F$ function to determine the Kähler metric of the manifold $\mathcal{M}_{\text {Kahler }}$. This $F$ function can be used to determine the Kähler potential $\mathcal{K}$ and the metric $G_{A \bar{B}} \equiv-\mathcal{K}_{A \bar{B}}=-\partial_{A} \bar{\partial}_{B} \mathcal{K}$ in the following way [6], 40]:

$$
\mathcal{K}(Z, \bar{Z})=\ln \left(Z^{I} N_{I J} \bar{Z}^{J}\right) \quad \text { with } \quad N_{I J}=i\left(\partial_{I} \partial_{J} F-\bar{\partial}_{I} \bar{\partial}_{J} F\right)
$$

where $Z^{I}=\frac{X^{I}}{X^{1}} \equiv\left\{1, Z^{A}\right\}$ and the Kähler metric therefore is the usual form $d s^{2}=$ $-\mathcal{K}_{A \bar{B}} d Z^{A} d \bar{Z}^{B}$. Observe that the metric is only positive definite in the region where $Z^{I} N_{I J} \bar{Z}^{J}$ is positive definite. Therefore $\mathcal{K}_{A \bar{B}}$ is negative definite [6], 40].

It is now time to use the power of the $c$-map to determine the quaternionic metric for our case. To build the quaternionic manifold we need $4(n+1)$ coordinates. The $Z^{A}, \bar{Z}^{A}$ contribute $2 n$ coordinates. The other $2 n$ coordinates are denoted as $A^{I}, B_{I}$, along with

we also know that these instantons exist. Therefore in this paper we will try to give as much information as possible, for these instantons, that do not rely on explicit lagrangian formulations. In the sequel to this paper we will attempt more explicit constructions. 
two more complex coordinates $\phi, \varphi$. The $c$-map then defines the quaternionic metric in the following way 6 [20:

$$
d s^{2}=|d \phi|^{2}-2 e^{-\phi}(\operatorname{Re} \mathcal{N})_{I \bar{J}} W^{I} \bar{W}^{J}+e^{-2 \phi}\left|d \varphi-\frac{A \cdot d B-B \cdot d A}{2}\right|^{2}-4 \mathcal{K}_{A \bar{B}} d Z^{A} d \bar{Z}^{B}
$$

where it should be clear that the Kähler geometry (3.27) forms a submanifold in the quaternionic space as expected. The structure of the universal hypermultiplet can also be extracted from (3.29). The components of the matrix $\mathcal{N}$, and $W^{I}$ are defined as:

$$
\mathcal{N}_{I J}=-i \partial_{\bar{I}} \partial_{\bar{J}} \bar{F}-\frac{N_{I K} N_{J L} X^{K} X^{L}}{X^{I} N_{I J} X^{J}}, \quad W^{I}=\left[(\operatorname{Re} \mathcal{N})^{-1}\right]^{I J}\left(2 \overline{\mathcal{N}}_{J K} d A^{K}-i d B_{J}\right)
$$

where $\operatorname{Re} \mathcal{N}$ is negative definite. For other details about the properties of $\mathcal{N}$ etc the readers may want to refer to [6], [41], [16], 40]. In the remaining part of this section we will give an explicit realisation of the quotient space (3.18).

\section{$\underline{\text { Realisation of the quotient space }}$}

To give an explicit realization of the homogeneous space (3.18) i.e $\frac{G_{2}}{S p(1) \times S p(1)} \equiv \frac{G_{2}}{S O(4)}$, we use the embedding of the exceptional complex Lie group $G_{2}(\mathbf{C})$ into the complex orthogonal Lie group $S O(7, \mathbf{C})$. Similar embeddings are valid for the two real forms of $G_{2}$, since the compact group $G_{2}^{C}(\mathbf{R})$ is included in $S O(7, \mathbf{R})$ and the non-compact real group $G_{2}^{N C}(\mathbf{R})$ in the real Lie group $S O(4,3)$. In the following, we will consider only the complex case and so we will omit the presence of $\mathbf{C}$ in the definition of our Lie groups.

The group $G_{2}$ has been shown [42], 443] to be isomorphic to the group of orthogonal transformations $S O(7)$ acting on the vector space $\mathbf{C}^{7}$ and leaving invariant a third-order completely antisymmetric tensor $T$. It is completely characterized by the following:

$$
T_{127}=T_{154}=T_{163}=T_{235}=T_{264}=T_{374}=T_{576}=1
$$

Choosing to realize the group $S O(7)$ by matrices $G \equiv\left\{g_{a b}\right\} \in \mathbf{C}^{7 \times 7}$ with determinant equal to 1 that satisfy the orthogonality relation:

$$
G^{\top} G=I \Longleftrightarrow g_{a b} g_{a c}=\delta_{b c}
$$

20 We are using the notations of 40 . 
we know that $G$ will thus be charaterized by 21 independent parameters. The invariance of the tensor $T$ under such transformations may be written as

$$
G^{\top} T_{a} G=g_{a b} T_{b} \Longleftrightarrow T_{a e f} g_{e c} g_{f d}=g_{a b} T_{b c d}
$$

where $T_{a}$ is the $7 \times 7$ matrix which elements are given by $\left(T_{a}\right)_{b c}=T_{a b c}$. It gives rise to 7 additional constraints on the elements of $G$ and $G$ thus contains the 14 independent parameters that leads to $G_{2}$.

A simple realization of these conditions could be easily seen when we consider the algebra $g_{2}$. It can indeed be realized as the set of orthogonal matrices $M \in o(7)$ such that $M^{\top}=-M$ and satisfying the invariance condition

$$
\left[T_{i}, M\right]=a_{i j} T_{i}
$$

which can be easily obtained from the relation (3.33) using the usual derivation of the exponential map which relates the group and algebra elements. We thus find an explicit form of $M \in G_{2}$ in terms of 14 independent parameters as:

$$
\left(\begin{array}{ccccccc}
0 & a_{12} & a_{13} & a_{14} & a_{15} & a_{16} & a_{17} \\
-a_{12} & 0 & a_{23} & a_{24} & a_{25} & a_{26} & a_{27} \\
-a_{13} & -a_{23} & 0 & a_{34} & a_{35} & a_{36} & -a_{15}-a_{26} \\
-a_{14} & -a_{24} & -a_{34} & 0 & a_{27}-a_{36} & -a_{17}+a_{35} & a_{16}-a_{25} \\
-a_{15} & -a_{25} & -a_{35} & -a_{27}+a_{36} & 0 & a_{12}-a_{34} & a_{13}+a_{24} \\
-a_{16} & -a_{26} & -a_{36} & a_{17}-a_{35} & -a_{12}+a_{34} & 0 & -a_{14}+a_{23} \\
-a_{17} & -a_{27} & a_{15}+a_{26} & -a_{16}+a_{25} & -a_{13}-a_{24} & a_{14}-a_{23} & 0
\end{array}\right)
$$

Let us mention that the maximal subalgebra $s o(4)=s u(2) \oplus s u(2)$ is easily identified. Indeed, we first take $a_{i 5}=a_{i 6}=a_{i 7}=0$ for $i=1,2,3$ to reduce the matrix to the form

$$
\left(\begin{array}{ccccccc}
0 & a_{12} & a_{13} & a_{14} & 0 & 0 & 0 \\
-a_{12} & 0 & a_{23} & a_{24} & 0 & 0 & 0 \\
-a_{13} & -a_{23} & 0 & a_{34} & 0 & 0 & 0 \\
-a_{14} & -a_{24} & -a_{34} & 0 & 0 & 0 & 0 \\
0 & 0 & 0 & 0 & 0 & a_{12}-a_{34} & a_{13}+a_{24} \\
0 & 0 & 0 & 0 & -a_{12}+a_{34} & 0 & -a_{14}+a_{23} \\
0 & 0 & 0 & 0 & -a_{13}-a_{24} & a_{14}-a_{23} & 0
\end{array}\right)
$$


and then take the six remaining independent parameters as $a_{34} \pm a_{12}=2 x_{ \pm 3}, a_{24} \mp a_{13}=$ $2 x_{ \pm 2}, a_{14} \pm a_{23}=2 x_{ \pm 1}$ to get the direct sum decomposition as $\mathbf{A} \oplus \mathbf{B}$, where:

$$
\begin{aligned}
\mathbf{A}=\left(\begin{array}{ccccccc}
0 & x_{+3} & -x_{+2} & x_{+1} & 0 & 0 & 0 \\
-x_{+3} & 0 & x_{+1} & x_{+2} & 0 & 0 & 0 \\
x_{+2} & -x_{+1} & 0 & x_{+3} & 0 & 0 & 0 \\
-x_{+1} & -x_{+2} & -x_{+3} & 0 & 0 & 0 & 0 \\
0 & 0 & 0 & 0 & 0 & 0 & 0 \\
0 & 0 & 0 & 0 & 0 & 0 & 0 \\
0 & 0 & 0 & 0 & 0 & 0 & 0
\end{array}\right) \\
\mathbf{B}=\left(\begin{array}{ccccccc}
0 & -x_{-3} & x_{-2} & x_{-1} & 0 & 0 & 0 \\
x_{-3} & 0 & -x_{-1} & x_{-2} & 0 & 0 & 0 \\
-x_{-2} & x_{-1} & 0 & x_{-3} & 0 & 0 & 0 \\
-x_{-1} & -x_{-2} & -x_{-3} & 0 & 0 & 0 & 0 \\
0 & 0 & 0 & 0 & 0 & -2 x_{-3} & 2 x_{-2} \\
0 & 0 & 0 & 0 & 2 x_{-3} & 0 & -2 x_{-1} \\
0 & 0 & 0 & 0 & -2 x_{-2} & 2 x_{-1} & 0
\end{array}\right)
\end{aligned}
$$

We also see the inclusion of the preceding subalgebra $s o(4)$ of $G_{2}$ in the algebra $s o(4) \oplus s o(3)$ as a subalgebra of $s o(7)$.

\section{Coordinates of the quotient space}

We start with the well-known realization of the Grassmannian of nondegenerate threeplanes $\mathrm{Gr}_{4}\left(\mathbf{C}^{7}\right)$ which is isomorphic to $S L(7) / \operatorname{Aff}(4,3)$ where $\operatorname{Aff}(4,3)$ is realized by matrices of the form

$$
\begin{aligned}
& G_{0}=\left(\begin{array}{cc}
G_{11} & 0 \\
G_{21} & G_{22}
\end{array}\right), \quad G_{11} \in \mathbf{C}^{4 \times 4}, \quad G_{22} \in \mathbf{C}^{3 \times 3} \\
& G_{21} \in \mathbf{C}^{3 \times 4}, \quad \operatorname{det} G_{11} \cdot \operatorname{det} G_{22}=1
\end{aligned}
$$

We then define homogeneous coordinates on $G r_{4}\left(\mathbf{C}^{7}\right)$ as

$$
\mathcal{X}=\left(\begin{array}{c}
X \\
z^{\top} \\
Y
\end{array}\right), \quad X, Y \in \mathbf{C}^{3 \times 3}, \quad z \in \mathbf{C}^{3}
$$


so that $S L(7)$ acts from the left as $\mathcal{X}^{\prime}=G \mathcal{X}$ with $G \in S L(7)$ and $\operatorname{Aff}(4,3)$ is thus the isotropy group of the origin chosen as $\mathcal{X}_{0}=\left(0,0, I_{3}\right)^{\top}$ and $\mathcal{X}=G \mathcal{X}_{0}$. The restriction to $S O(7)$ leads to the isotropy group $S O(4) \otimes S O(3)$ since $G_{0}$ being orthogonal, it implies $G_{21}=0$. The homogeneous coordinates $\mathcal{X}=G \mathcal{X}_{0}$ of $S O(7) /(S O(4) \times S O(3))$ satisfy the orthogonality condition:

$$
X^{\top} X+z z^{\top}+Y^{\top} Y=1
$$

which represents a set of 6 independent equations between the 21 parameters characterizing $\mathcal{X}$. Since we have

$$
\operatorname{dim}\left[\frac{S L(7)}{\operatorname{Aff}(4,3)}\right]=\operatorname{dim}\left[\frac{S O(7)}{S O(4) \times S O(3)}\right]=12
$$

the usual way to reduce further the independent quantities is to use the affine coordinates defined as

$$
W=X Y^{-1}, \quad w=z^{\top} Y^{-1}, \quad \operatorname{det} Y \neq 0
$$

Let us now consider the quotient space $G_{2} / S O(4)$. We have

$$
\operatorname{dim}\left[\frac{G_{2}}{S O(4)}\right]=14-6=8
$$

This space can be characterized by the homogeneous coordinates $\mathcal{X}=G \mathcal{X}_{0}$ where now $G \in G_{2} \subset S O(7)$ and thus satisfies the relations (3.33). They give rise to supplementary conditions on the 21 parameters characterizing $\mathcal{X}$. Indeed, we can write

$$
\mathcal{X}=G \mathcal{X}_{0}=G\left(\begin{array}{c}
0 \\
0 \\
I_{3}
\end{array}\right)=\left(\begin{array}{ccc}
g_{15} & g_{16} & g_{17} \\
g_{25} & g_{26} & g_{27} \\
g_{35} & g_{36} & g_{37} \\
g_{45} & g_{46} & g_{47} \\
g_{55} & g_{56} & g_{57} \\
g_{65} & g_{66} & g_{67} \\
g_{75} & g_{76} & g_{77}
\end{array}\right)
$$

The relations (3.33) imply, together with (3.31), that:

$$
g_{a b}\left(T_{b}\right)_{76}=g_{a 5}=T_{a e f} g_{e 7} g_{f 6}
$$


so the 7 parameters of the first column of $\mathcal{X}$ are expressed in terms those of the other columns. Moreover, we have the orthogonality condition (3.40) which implies 3 more relations between the remaining parameters:

$$
g_{a 6} g_{a 6}=g_{a 7} g_{a 7}=1, \quad g_{a 6} g_{a 7}=0
$$

So, the number of independent parameter has been reduced to 11 at this stage. As before, the last step to reduce further the number of parameters is to use the affine coordinates. The conditions on $W$ and $w$ that leads a characterization of the quotient $G_{2} / S O(4)$ are explicitly given in 44].

With this we are now ready to discuss the magic square. We will also show how some of the aspects that we studied here can be elucidated from the properties of the magic square.

\section{On the classification of quaternionic manifolds: the magic square}

The magic square in mathematics is used to show the relation between division algebras, Jordan algebras [12] and Lie algebras. The idea was first developed by Freudenthal, Rozenfeld and Tits [7] and is introduced to string theory by Gunaydin-Sierra-Townsend [4]. The magic square in mathematics is a $4 \times 4$ square with the entries given by elements of the Lie algebras. The columns of the magic square are defined by the Jordan algebras, whereas the rows are defined by the division algebras [45]. The division algebras are the real $(\mathbf{R})$, complex $(\mathbf{C})$, quaternion $(\mathbf{Q})$ and the octonion $(\mathbf{O})$. The columns are labelled by: $J^{3}(\mathbf{R}), J^{3}(\mathbf{C}), J^{3}(\mathbf{Q}), J^{3}(\mathbf{O})$ where $J^{3}(\mathbf{K})$ is the algebra of $3 \times 3$ Hermitian matrices over $\mathbf{K}$. The magic square is then given by:

\begin{tabular}{|l|l|l|l|}
\hline$A_{1}$ & $A_{2}$ & $C_{3}$ & $F_{4}$ \\
\hline$A_{2}$ & $A_{2}^{2}$ & $A_{5}$ & $E_{6}$ \\
\hline$C_{3}$ & $A_{5}$ & $D_{6}$ & $E_{7}$ \\
\hline$F_{4}$ & $E_{6}$ & $E_{7}$ & $E_{8}$ \\
\hline
\end{tabular}

where $A_{i}, C_{i}, D_{i}, E_{i}, F_{4}$ are the usual $S U, S p, S O, E_{6,7,8}$ and $F_{4}$ Lie groups respectively (a similar square can be drawn for the corresponding algebras also). The rules for filling up the entry $L$ of the magic square can be given by the relation (see for example [45]):

$$
L=\operatorname{Der} A \oplus\left(A_{0} \otimes J_{0}\right) \oplus \operatorname{Der} J
$$


where Der $A$ and Der $J$ are the generators of the automorphism group of the Hurwitz (division) algebra $A$ and of the algebra $J, A_{0}$ are the pure imaginary elements of $\mathbf{R}_{0}=$ $S^{0}, \mathbf{C}_{0}=S^{1}, \mathbf{Q}_{0}=S^{3}$ and $\mathbf{O}_{0}=S^{7}$ and $J_{0}$ are the elements of trace zero of the Jordan algebra $J$. To make this clear, we can write the magic square in terms of the dimensions of the Lie algebras in the following way:

\begin{tabular}{|c|c|c|c|}
\hline 3 & 8 & 21 & 52 \\
\hline 8 & 16 & 35 & 78 \\
\hline 21 & 35 & 66 & 133 \\
\hline 52 & 78 & 133 & 248 \\
\hline
\end{tabular}

The reason for the magical property of the square can be made clear from the entry-rule given in (4.1). In terms of last to the first row, we can write the elements of the magic square in the following way:

\begin{tabular}{|r|l|l|l|}
\hline 52 & $78=52+1 \times 26$ & $133=52+3 \times 26+3$ & $248=52+7 \times 26+14$ \\
\hline 21 & $35=21+1 \times 14$ & $66=21+3 \times 14+3$ & $133=21+7 \times 14+14$ \\
\hline 8 & $16=8+1 \times 8$ & $35=8+3 \times 8+3$ & $78=8+7 \times 8+14$ \\
\hline 3 & $8=3+1 \times 5$ & $21=3+3 \times 5+3$ & $52=3+7 \times 5+14$ \\
\hline
\end{tabular}

For more details see for example [46] (and references therein). The interesting feature of the magic square is that its symmetric and four of the five exceptional Lie algebras occur in the last row. In fact one could also add $G_{2}$ to the magic square by adding an extra column (therefore some literature also refers the magic square as a $4 \times 5$ rectangle). The extra column corresponds to the Jordan algebra $\mathbf{R}$ (see figure below):

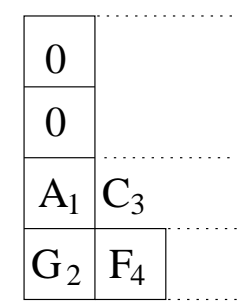

where other elements of the square are to be filled in the dotted parts. Once we have the Lie groups, we should ask how to accomodate the quaternionic spaces or Kähler spaces in the magic square. To describe this let us use the column containing $G_{2}$ and $A_{1}$ Lie groups as this is the simplest. In the language of constrained instantons, observe that in 
the maximal subgroup of $G_{2}$ i.e $S U(2) \times S U(2)$ one of the $S U(2)$ is gauged. This leaves one free $S U(2)$ and the quaternionic manifold is (3.18) (or (3.26) in the non-compact limit). For the next element of the magic square i.e $A_{1}$ here, we look at the $U(1)$ subgroup of the ungauged $S U(2)$ and gauge it. The resulting space is $\frac{S U(2)}{U(1)}$ or $\frac{S U(1,1)}{U(1)}$ in the non-compact limit. This reproduces the next element of the magic square. Finally since we have gauged the remaining $U(1)$ we have nothing else to gauge, so the other two remaining elements of the magic square are 0 and 0 (see figure above).

Observe however that in the above figure we have ignored a subtlety regarding the $c$-map for the $G_{2}$ case. This has to do with the existence of two different non-trivial $F$ functions for the corresponding $S U(1,1) / U(1)$ Kähler space [6], [5]. This can be illustrated in the following way:

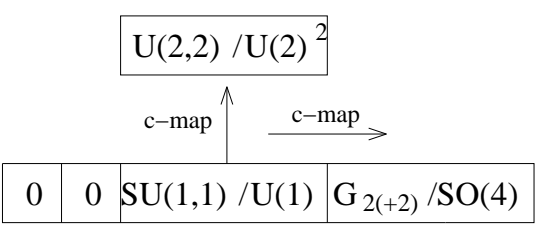

where we see that the same Kähler space can give rise to two different quaternionic space. One of the quaternionic space doesn't lie in the magic square and is generated by a $F$ function given by:

$$
F\left(X^{I}\right)=\left(X^{1}\right)^{2}-\left(X^{2}\right)^{2}
$$

The fact that this is no contradiction is explained in [6]. What we are looking for is the c-map related to Jordan algebra and this is given by the horizontal arrow.

Thus for the generic case our procedure should now be clear. We are gauging various subgroups as we move along the magic square. We call this sequential gauging. Let us consider a part of magic square represented by non-compact group elements $A, B, C$ and $D$ in the following way:

\begin{tabular}{|l|l|l|l|}
\hline $\mathrm{D}$ & $\mathrm{C}$ & $\mathrm{B}$ & $\mathrm{A}$ \\
\hline
\end{tabular}

Question now is whether we can determine the corresponding manifolds associated with these elements of the magic square using the arguments of constrained instantons. The manifold associated with group $A$ is easy. This has to be a quaternionic manifold in such a way that a $S U(2)$ subgroup of the maximal group is gauged. What is the maximal subgroup of $A$ here? This is exactly given by the next element $B$ of the magic square. Let 
$B_{c}$ be the compact version of the group $B$. Then the maximal subgroup of $A$ is clearly $B_{c} \times S U(2)$ giving rise to the quaternionic manifold:

$$
\frac{A}{B_{c} \times S U(2)}
$$

Now question is whether we can determine the next manifold that should be Kähler (recall the $c$-map constraint). Looking at the next element we find the group $C$ whose compact version is $C_{c}$. What we need now is that the ungauged group $B$ should decompose into $C_{c}$ and another subgroup. This is easy to determine from the list of subgroups given in [38]. Let the subgroup be $H_{1}$. This therefore gives us the Kähler manifold:

$$
\frac{B}{C_{c} \times H_{1}}
$$

whose $c$-map therefore will be (4.3). Going in this way we can reproduce all the manifolds

\begin{tabular}{|c|c|c|c|}
\hline (D) & (C) $\ldots$ & (B) & A \\
\hline $\mathrm{H}_{3}$ & (Dc) $\mathrm{x}_{\mathrm{H}}$ & (C) $\mathrm{X}^{2} \mathrm{H}_{1}$ & Bc $\times S U(2)$ \\
\hline
\end{tabular}
associated with the elements of the magic square in the following way:

where the subgroups $H_{i}$ could in principle be determined from [38]; and the dotted lines are used to show the connection between the ungauged groups. But the story doesn't end here because it turns out that the subgroups themselves are not arbitrary. The quaternionic space was determined by gauging the $S U(2)$ subgroup. This was related to the constrained instantons. Now what could be the next subgroup that we can gauge? Clearly this has to be a $U(1)$ subgroup related to semilocal strings. Similarly we can ask about the next to next subgroup. Since we gauged $S U(2)$ as well as $U(1)$ we cannot gauge any other group! So our prediction for the magic square will be

$$
H_{1}=U(1), \quad H_{2}=1, \quad H_{3}=1
$$

Observe however that there are some subtleties related to these identifications because the third manifold associated with the group $C$ in the magic square should be a real manifold, so we might have to consider appropriate complex conjugates of the relevant groups. The final picture that emerges from all the above consideration is: 


\begin{tabular}{|c|l|l|l|}
\hline $\mathrm{D}$ & $\frac{\mathrm{C}}{\mathrm{D}_{\mathrm{c}}}$ & $\frac{\mathrm{B}}{\mathrm{C}_{\mathrm{c}} \times \mathrm{U}(1)}$ & $\frac{\mathrm{A}}{\mathrm{B}_{\mathrm{c}} \mathrm{x}} \mathrm{SU}(2)$ \\
\hline
\end{tabular}

which we would verify in the next few examples. A more detailed analysis of the manifolds other than the quaternionic ones will be presented in the sequel. In the following sections we will mainly study the quaternionic manifolds associated with $E_{n}$ and $F_{4}$ groups.

\section{1. $E_{6}$ quaternionic space}

Our first case is to look for a theory with global symmetry $\mathcal{G}=E_{6}$. To extract the quaternionic space associated with this group we should study the maximal subalgebra 21. The maximal regular subalgebra of $E_{6}$ can be extracted from the extended Dynkin diagram:

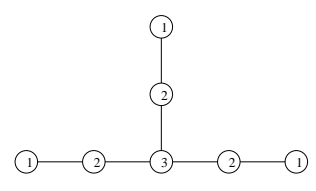

and is given by $\mathcal{H}=s u(6) \oplus s u(2)$. This immediately tells us two things: One, we are dealing with a gauge theory with $\mathcal{H}_{1}=S U(2)=S p(1)$ gauge group, and two, the manifold $\mathcal{M}_{E_{6}}$ is

$$
\mathcal{M}_{E_{6}}=\frac{E_{6}}{S U(6) \times S p(1)} .
$$

From the analysis that we presented in the previous section and using [37], one can verify that $\pi_{3}\left(\frac{E_{6}}{S U(6)}\right)=1$, so we need to gauge an $S U(2)$ subgroup. Indeed, as like the previous

21 Notice that in addition to the choice of maximal subalgebras, we also ask for symmetric subalgebras of the groups. The symmetric subalgebras for various groups have been listed in [38]. For the $A_{n}, B_{n}, C_{n}, D_{n}$ cases, they are

$$
\begin{gathered}
s u(p+q) \rightarrow \operatorname{su}(p) \oplus s u(q) \oplus u(1), \quad s o(p+q) \rightarrow s o(p) \oplus s o(q) \\
s p(2 p+2 q) \rightarrow s p(2 p) \oplus s p(2 q)
\end{gathered}
$$

where $p$ and $q$ form the various distribution (as even or odd integers). For the $E_{n}$ cases one would have

$$
\begin{aligned}
& e_{8} \rightarrow s o(16), \quad s u(2) \oplus e_{7} \\
& e_{7} \rightarrow s u(8), \quad s u(2) \oplus s o(12), \quad e_{6} \oplus u(1) \\
& e_{6} \rightarrow s p(8), \quad s u(2) \oplus s u(6), \quad s o(10) \oplus u(1), \quad f_{4} .
\end{aligned}
$$

From the list one has to extract out the relevant algebras that we would require for our case. 
cases, one can find the following decomposition:

$$
27 \rightarrow(\overline{6}, 2)+(15,1)
$$

under $S U(6) \times S U(2)$ subgroup. The $S U(2)$ subgroup that we want to gauge is slightly different. This subgroup is the diagonal subgroup of the $S U(2)_{g} \times S U(2)_{l}$ where $g, l$ stand for the global and local groups respectively. Both the global and the local groups are broken by Higgs expectation value - once we give a VEV to $(\overline{\mathbf{6}}, \mathbf{2})$ - and therefore an $S U(2)_{g}^{\prime}$ group survives (which we will call $S U(2)$ henceforth). Since $S U(2) \sim S^{3}$, the homotopy classification will tell us that $\pi_{3}\left(S^{3}\right)=\mathbf{Z}$. These are the constrained instantons, and therefore should have a construction via the quaternion as we discussed before. These instantons are again non-trivially fibered over the space (4.8) and therefore exist only as semi-local defects.

Thus we seem to get our required exceptional semilocal defect in this model. However in the process of deriving this we have ignored a subtlety. This subtlety cannot be seen at the level of group structure, in the sector of Seiberg-Witten theory that we study, but is visible when we look at the corresponding Seiberg-Witten curve associated to our manifold. Therefore let us construct the corresponding curve by modifying the $G_{2}$ curve that we discussed in (3.20). The reason why we want to start from $G_{2}$ and go all the way to $E_{8}$ is because of the last row of the magic square

$$
\begin{array}{|l|l|l|l|l|}
\hline \mathrm{G}_{2} & \mathrm{~F}_{4} & \mathrm{E}_{6} & \mathrm{E}_{7} & \mathrm{E}_{8} \\
\hline
\end{array}
$$

which is expressed as a part of the $4 \times 5$ rectangle. Since the magic square elements are related, we will then take (3.20) and add changes so that it eventually becomes the curve for $E_{6}$, and then subsequently for other cases (we have ignored the $F_{4}$ case for the time being because it will be shown later to be very close to the $E_{6}$ case).

Our first modification would be to change the powers of $z$ in (3.20). This modifies the curve to the following:

$$
\begin{gathered}
\left(y+\frac{12 a_{1} z x-4 a_{1} a_{2} z^{3}-4 a_{1}^{2} z^{2}+12 a_{3} z^{2}}{24}\right)^{2}=x^{3}-\frac{x}{48}\left[\left(a_{1}^{4}+8 a_{1}^{2} a_{2}+16 a_{2}^{2}\right) z^{4}+\right. \\
\left.-24\left(a_{1} a_{3}+2 a_{4}\right) z^{3}\right]+\frac{1}{864}\left[a_{1}^{8} z^{8}+\left(12 a_{1}^{4} a_{2}+48 a_{1}^{2} a_{2}^{2}+64 a_{2}^{3}\right) z^{6}+216 a_{3}^{2} z^{4}+\right. \\
\left.-\left(36 a_{1}^{3} a_{3}+72 a_{1}^{2} a_{4}+144 a_{1} a_{2} a_{3}+288 a_{2} a_{4}-864 a_{6}\right) z^{5}\right]
\end{gathered}
$$


with $a_{i}$ arbitrary. To fix the values of $a_{i}$ we have to study the singularity structures carefully. The discriminant locus of this equation near the points $z=0$ can be easily worked out. For us this will be given by

$$
\Delta \sim z^{8}+\mathcal{O}\left(z^{9}\right)
$$

up to an overall numerical factor. To study the singularities at $z \neq 0$ the curve (4.10) is not generic enough. To derive the actual curve we need to manipulate (4.10) further. We will do this in few steps. First observe that (4.10) can be re-written as:

$$
Y^{2}=x^{3}-x z^{3}(A z+B)+\frac{z^{4}}{864}\left(C z^{4}+D z^{3}+E z+F\right)
$$

where the new coefficients $A, \ldots, F$ and $Y$ are defined from (4.10) in the following way:

$$
\begin{aligned}
& Y=y+\frac{12 a_{1} z x-4 a_{1} a_{2} z^{3}-4 a_{1}^{2} z^{2}+12 a_{3} z^{2}}{24}, \quad C=a_{1}^{8}, \quad F=216 a_{3}^{2} \\
& A=a_{1}^{4}+16 a_{2}^{2}+8 a_{1}^{2} a_{2}, \quad D=12 a_{1}^{4}+48 a_{1}^{2} a_{2}^{2}+64 a_{2}^{3} \\
& B=-24\left(a_{1} a_{3}+2 a_{4}\right), \quad E=36 a_{1}^{3} a_{3}+72 a_{1}^{2} a_{4}+144 a_{1} a_{2} a_{3}+288 a_{2} a_{4}-864 a_{6}
\end{aligned}
$$

Secondly, that the curve (4.12) doesn't fully capture the $E_{6}$ singularities completely can be easily demonstrated (see also [47]). The dimensionality of $x, Y, z$ etc. can be worked out from the equation

$$
\frac{d \lambda_{\mathrm{SW}}}{d z}=\frac{d x}{Y}
$$

where $\lambda_{\mathrm{SW}}$ is the Seiberg-Witten differential. We can then break the $E_{6}$ global symmetry to $S O(10) \times U(1)$ such that the fundamental $\mathbf{2 7}$ decomposes as

$$
27=16_{+1}+10_{-2}+\mathbf{1}_{+4}
$$

where the subscripts denote the $U(1)$ charges. This would then imply that the coefficient of $x$ in (4.12) should have a $z^{2}$ term [47]. Similar conclusion can be extracted by further breaking the global symmetry to $D_{4} \equiv S O(8)$ where we know that $z^{2}$ should exist (see eq. (2.16) in 48). Therefore if we redefine $x, Y$ to $\tilde{x}, \tilde{y}$ as:

$$
\tilde{x}=x z^{-\frac{3}{2}}, \quad \tilde{y}=Y z^{-2}
$$


where the redefinition makes sense because we are not analysing the $z=0$ points, then (4.12) can be written as

$$
\tilde{y}^{2}=\tilde{x}^{3}-\tilde{x}\left(G z^{2}+A^{\prime} z+B\right)+\frac{1}{864}\left(C z^{4}+D z^{3}+E z+F\right)\left(1-\frac{1}{2} \log z+\ldots\right)
$$

where $A^{\prime}$ and $G$ are the minimal changes to (4.12). Observe that we can assume $A^{\prime} \propto A$ without a loss of generality.

The new curve (4.17) is almost the one discussed in 47] with the exception of the additional $\log z$ terms. These terms could be ignored for our case as we want to realise the pure $E_{6}$ global symmetry22. To complete the picture we need to derive the explicit form for $G, A^{\prime}$ and $a_{i}(i=1,2,3,4,6)$. These are given in terms of $E_{6}$ Casimirs defined in the following way [50]:

$$
p_{n}\left(x_{j}\right)=\sum_{\left\{n_{i}\right\}} \mathcal{C}_{\left\{n_{i}\right\}} x_{1}^{n_{1}} x_{2}^{n_{2}} x_{4}^{n_{3}} x_{5}^{n_{4}} x_{6}^{n_{5}} x_{8}^{n_{6}}
$$

where the operators $x_{i}$ are defined in terms of the Cartan subalgebra of $E_{6}$ and $n, n_{i}$ are integers satisfying the following algebraic equation:

$$
n \equiv\{2,5,6,8,9,12\}=n_{1}+2 n_{2}+4 n_{3}+5 n_{4}+6 n_{5}+8 n_{6}
$$

and $\mathcal{C}_{\left\{n_{i}\right\}}$ are integers. The sum is over all possible integer solutions of the above equation (4.19). As an example the Casimir $p_{6}$ will be defined via the following values of the coeffcients $\mathcal{C}_{\left\{n_{i}\right\}}$ :

$$
\begin{array}{lll}
\mathcal{C}_{000010}=-1, & \mathcal{C}_{410000}=-1062, & \mathcal{C}_{011000}=\frac{5}{4},
\end{array}
$$

where one can get the full list in [50]. Using these Casimirs one can easily determine the coefficients $G, A^{\prime}$ and $a_{i}$ by comparing the curve (4.17) with the one given in [47. They

22 It is not clear to us what singularities would the additional $\log z$ dependent terms would imply. Of course additional singularities besides $E_{6}$ have been observed for certain F-theory curves in 49, but there the singularities were simple. 
are given by:

$$
\begin{aligned}
& G=-\frac{p_{2}}{3}, \quad A^{\prime}=\frac{2 p_{5}}{3}, \quad a_{1}=2^{5 / 8} 3^{3 / 8} \approx 2.328 \\
& \frac{a_{3}^{2}}{4}=\frac{32}{135} p_{12}-\frac{298}{18225} p_{2}^{2} p_{8}-\frac{101}{218700} p_{2}^{3} p_{6}+\frac{13}{405} p_{6}^{2}-\frac{49}{1049700} p_{2}^{6}-\frac{19}{3645} p_{2} p_{5}^{2} \\
& a_{4}=\frac{1}{2}\left[\frac{7}{10368} p_{2}^{4}-\frac{11}{1080} p_{2} p_{6}+\frac{p_{8}}{45}-2^{5 / 8} 3^{3 / 8} a_{3}\right] \\
& a_{2}=\omega+\frac{b^{2}}{9 a^{2}} \cdot \frac{1}{\omega}-\frac{b}{3 a} \approx \omega+\frac{1.837}{\omega}-1.355 \\
& a_{6}=\frac{a_{1}^{3} a_{3}}{24}+\frac{a_{1}^{2} a_{4}}{12}+\frac{a_{1} a_{2} a_{3}}{6}+\frac{a_{2} a_{4}}{3}-\frac{p_{2}^{2} p_{5}}{18}-\frac{8}{21} p_{9}
\end{aligned}
$$

where using

$$
c=576 p_{6}-56 p_{2}^{3}-144 \sqrt{6}, \quad a=64, \quad b=2^{21 / 4} 3^{7 / 4} \approx 260.237
$$

we can define $\omega$ appearing in the defination of $a_{2}$ above as

$$
\omega^{3}=-\frac{1}{2}\left(\frac{2}{27} \frac{b^{3}}{a^{3}}-\frac{c}{a}\right) \pm \frac{1}{2} \sqrt{\left(\frac{2}{27} \frac{b^{3}}{a^{3}}-\frac{c}{a}\right)^{2}-4\left(\frac{b}{3 a}\right)^{6}}
$$

which would imply that $a_{2}$ is a negative definite quantity. From the above we can also determine the proportionality constant between $A$ and $A^{\prime}$. This is given by

$$
\frac{2 p_{5}}{48 a_{2}^{2}+2^{17 / 4} 3^{7 / 4} a_{2}+36 \sqrt{6}} \approx \frac{2 p_{5}}{48 a_{2}^{2}+302.8 a_{2}+29.37}
$$

where $a_{2}$ can be extracted from above. This therefore completes the full analysis of the Seiberg-Witten curve for the system.

The subtlety that we were alluding to earlier lies in the realisation of the subalgebra (or the subgroup (4.9)) associated with the $E_{6}$ symmetry that would be used to determine the quaternionic manifold directly from the curve (4.17). Knowing the discriminant we can in principle extract the corresponding subalgebra associated with the global group $\mathcal{G}=E_{6}$ provided the background space is specified. However the issue is more intricate because:

- There is no lagrangian description of the system with exceptional global symmetry. In fact existence of the curve doesn't guarantee that the system is a SYM theory in some limit.

- Even if there exist some suitable description, the system is at strong coupling [49] where a controlled analytical calculation cannot be done. Furthermore due to large number of flavors the theory is not asymptotically free. 
All these issues might still be resolved if we embed our gauge theory in some stringy set-up. There are various possibilites here. We might embed it in a F-theory set-up much like the one discussed in [31], [51], [49], [52], [27], [24] etc. or in a M-theory set-up like 5323 . Using any of these cases, all we need is that the eight singularities decompose into a bunch of six and two singularities giving rise to the discriminant and subgroup

$$
\Delta \sim(z-a)^{6}\left(z^{2}+b\right) \Rightarrow E_{6} \subset S U(6) \times S U(2)
$$

which is of course the maximal subgroup for our case. Once the global symmetry is broken, a lagrangian description is possible when the system is embedded in a F-theory set-up. In F-theory, analysing the curve however leads to the following subalgebra:

$$
s u(5) \oplus s u(2) \oplus u(1)
$$

instead of the subalgebra associated with the decomposition (4.9). This is almost the maximal subalgebra that we wanted, but not quite 24 . In fact $s u(6)$ is broken to $s u(5) \oplus u(1)$. Thus this is the closest we come to getting the full structure of the coset space directly from type IIB string theory (or F-theory)25. In fact what we need is that the $\mathbf{6}$ of $S U(6)$

23 In fact, since our theory is just a sector of the Seiberg-Witten theory, all the subtleties afflicting the original theory will not have much effect on our analysis. Furthermore the SeibergWitten curve is the only output that we will be using for our case.

24 When our theory is embedded in the full Seiberg-Witten theory the same subtlety should show up in determining the Higgs branch. However in the absence of a proper lagrangian description this may not be easy to implement.

25 The full configuration on the other hand can be determined in the following way: First we decompose the $E_{6}$ adjoint in terms of the subalgebra (4.26) as

$$
\mathbf{7 8}=(\mathbf{2 4}, \mathbf{1})_{0}+(\mathbf{1}, \mathbf{1})_{0}+(\mathbf{1}, \mathbf{3})_{0}+(\mathbf{1 0}, \mathbf{2})_{-3}+(\mathbf{5}, \mathbf{1})_{6}+\text { c.c }
$$

where the subscripts refer to the $U(1)$ charges and the c.c are associated with $\overline{10}$ and $\overline{5}$ with $U(1)$ charges 3 and -6 respectively. Secondly, having given the decomposition, the rest of the discussion now should follow the familiar line developed in the series of papers [49], [39]. We will not elaborate on this aspect as the readers can look up the details in those papers. It'll simply suffice to mention that the non-trivial configuration required to get the full group structure lies in the process of brane creation via the Hanany-Witten effect [54 leading to strings with multiple prongs [55], [56], [57] that fill out the rest of the group generators [39]. 
should decompose under $S U(5) \times U(1)$ as:

$$
6 \rightarrow \mathbf{5}_{1}+\mathbf{1}_{-5}
$$

which would form the ungauged maximal subgroup. The associated monodromy matrix is then clearly

$$
\left(\begin{array}{ll}
-1 & -1 \\
-1 & -2
\end{array}\right)
$$

which leaves one of the dyonic point in the monodromy matrix and determines the rest of the $S U(6)$ generators non-perturbatively. The surviving diagonal $S U(2)$ is now gauged according to our earlier discussion 26 .

The above construction therefore gives us the constrained instanton configurations associated with global symmetry $E_{6}$ that are fibered over the quaternionic base $\mathcal{M}_{E_{6}}$ (4.8). However, as in the previous sections, this is not quite the manifold that we are looking for. We should aim for the non-compact version of (4.8) i.e

$$
V(1,2) \equiv \frac{E_{6(+2)}}{S U(6) \times S U(2)}
$$

where +2 in the bracket denote the difference between the number of compact and noncompact generators. The corresponding Kähler space associated with (4.30) can be constructed by gauging subgroups of $S U(6)$ according to our scheme. The relevant subgroup of $S U(6)$ for us is $S U(3) \times S U(3) \times U(1)$ under which $\mathbf{6}$ decomposes as:

$$
\mathbf{6}=(\mathbf{1}, \mathbf{3})_{-1}+(\mathbf{3}, \mathbf{1})_{+1}
$$

where by modding $A_{5}$ by the corresponding subgroup gives rise to the following Kähler space:

$$
\frac{S U(3,3)}{S U(3) \times S U(3) \times U(1)}
$$

where $S U(3,3)$ is the non-compact version of $S U(6)$. Observe also that (4.32) is exactly of the form (4.4) with $H_{1}=U(1)$ and $C_{c}=S U(3) \times S U(3)$. Furthermore, under a $c$-map (4.31) does give us (4.30) once the $F$-function is specified. We will specify the $F$-function a bit later. Looking now into the magic square for the $E_{6}$ sequence:

26 Recall that before combining the $S U(2)$ part of the unbroken global group with the local $S U(2)$ gauge symmetry we expect a monodromy matrix of the form $\left(\begin{array}{cc}3 & 2 \\ -2 & -1\end{array}\right)$. 


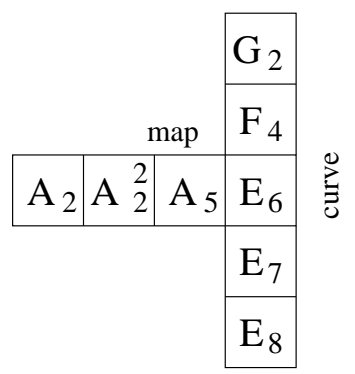

where the vertical sequence is shown to emphasise how the curves were constructed, and the horizontal sequence is constructed by various maps: $c, r$ etc., we can easily argue the various manifolds associated with the horizontal elements of the magic square using the technique of partial gauging of the subgroups discussed in the previous section. This will give us the following sequence:

\begin{tabular}{|c|c|c|c|}
\hline $\mathrm{SU} *(3)$ & $\frac{\mathrm{SL}(3 ; \mathrm{C})}{\mathrm{SU}(3)}$ & $\frac{\mathrm{SU}(3,3)}{\mathrm{SU}(3) \times \mathrm{U}(3)}$ & $\frac{\mathrm{E}_{6(+2)}}{\mathrm{SU}(6) \times \mathrm{SU}(2)}$ \\
\hline
\end{tabular}

where the third term in the sequence has $H_{2}=1$ and the fourth term has $H_{3}=1$ as predicted in (4.5). With this sequencing structure we can now determine the sigma-model metric associated with the constrained instantons fibered over the quaternionic base (4.10) (or (4.30) in the non-compact limit). The quaternionic metric is always of the form (3.29) which is derived from the corresponding Kähler metric (3.28). All we need to complete the picture for the $E_{6}$ case would be the $F$-value. We will present a detailed analysis of this in sec. 4.6 including a generic derivation for all possible cases.

Before we end this section, notice that we haven't yet checked whether there is some semilocal soliton that could be fibered over the space (4.32) much like the quaternionic examples studied so far. For this we have to study the associated vacuum structure. Whether this theory could be studied in the same moduli space as the present ones needs to be investigated. It is of course highly suggestive that there are semilocal string like defects because $\pi_{1}(U(1))=\mathbf{Z}$ and using the exact sequence for Lie group $\mathcal{G}$ and its subgroup $\mathcal{H}$ :

$$
0 \longrightarrow \pi_{2}\left(\frac{\mathcal{G}}{\mathcal{H}}\right) \longrightarrow \pi_{1}(\mathcal{H}) \longrightarrow \pi_{1}(\mathcal{G}) \longrightarrow \pi_{1}\left(\frac{\mathcal{G}}{\mathcal{H}}\right) \longrightarrow 0
$$

one can easily argue that for $\mathcal{G}=S U(n)=S U(6)$ and $\mathcal{H}=S U(3) \times S U(3)$ (or in fact for any generic Lie subgroups [58]):

$$
\pi_{1}\left(\frac{S U(6)}{S U(3) \times S U(3)}\right)=0=\pi_{2}\left(\frac{S U(6)}{S U(3) \times S U(3)}\right)
$$


showing that there could only be semilocal defects. We will however leave a detailed study of this for future investigations.

\section{2. $E_{7}$ quaternionic space}

Let us now turn towards the next group $\mathcal{G}=E_{7}$. The extended Dynkin diagram of $E_{7}$ :

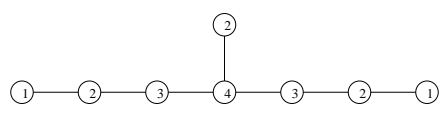

can be cut in different ways to give rise to various maximal regular subalgebras of $E_{7}$. They are given by

$$
s u(8), \quad \operatorname{spin}(12) \oplus s u(2), \quad s u(6) \oplus s u(3), \quad e_{6} \oplus u(1)
$$

where spin(12) actually comes from so(12) with some identification between the generators. From the set of steps that we mentioned earlier, we can immediately ignore the subalgebras $s u(8), s u(6) \oplus s u(3)$ and $e_{6} \oplus u(1)$ and therefore the associated groups $S U(8), S U(6) \times$ $S U(3), E_{6} \times U(1)$ as they cannot be realised in the present scenario (recall that the gauge group is $S U(2)$ 27. The above consideration immediately gives us the corresponding unique coset manifold for the global symmetry $E_{7}$ as

$$
\mathcal{M}_{E_{7}}=\frac{E_{7}}{\operatorname{Spin}(12) \times S p(1)} .
$$

Our previous consideration will require us to view this as a homogeneous quaternionic Kähler manifold. The $S U(2)$ constrained instantons are fibered over this manifold because the third homotopy group of the vacuum manifold is trivial i.e $\pi_{3}\left(\frac{E_{7}}{S O(12)}\right)=1$. But then again such a big global symmetry will not allow a lagrangian description of the system, so to make any concrete statements we have to analyse the maximal subgroup $S O(12) \times S U(2)$ associated with the system.

27 Observe however that the third homotopy groups of $S U(2)$ and $S U(3)$ are both given by

$$
\pi_{3}(S U(2))=\pi_{3}(S U(3))=\mathbf{Z}
$$

and therefore $S U(3)$ theory can also allow non-trivial constrained instantons. It would be interesting to study the manifold associated with this setup. 
However as before, analysing the corresponding Seiberg Witten curve will tell us that the actual subgroup realised perturbatively is different from $S O(12) \times S U(2)$ or $\operatorname{Spin}(12) \times$ $S p(1)$. To see this we will study the theory in few steps. Firstly, the breaking pattern for the $\mathbf{5 6}$ of $E_{7}$ is:

$$
56=(12,2)+(32,1)
$$

under $S O(12) \times S U(2)$. Giving a VEV to $(\mathbf{1 2}, \mathbf{2})$ the broken global $S U(2)$ can combine with the broken local $S U(2)$ and give us the unbroken global group $S U(2) \equiv S p(1)$. This is the $S p(1)$ that appears in (4.37). Furthermore once we have the coset space (4.37) we have to analyse the rest of the coset spaces from the magic square column:

$$
\begin{array}{|l|l|l|l|}
\hline \mathrm{C}_{3} & \mathrm{~A}_{5} & \mathrm{D}_{6} & \mathrm{E}_{7} \\
\hline
\end{array}
$$

To analyse the coset space (4.37) let us determine the curve associated with $E_{7}$ by deforming the $E_{6}$ curve (4.17) that we determined earlier. Our first attempt to determine the curve using the following values of the variables in (3.7):

$$
\{l, k, h, f, g\}=\left\{z, z^{2}, z^{3}, z^{3}, z^{5}\right\}
$$

can only tell us the discriminant behavior at $z=0$. To determine the curve at any generic point $z \neq 0$ we can deform (4.17) to the following curve:

$$
\tilde{y}^{2}=\tilde{x}^{3}-\tilde{x}\left(2 z^{3}+M z^{2}+N z+P\right)+\frac{1}{864}\left(Q z^{4}+R z^{3}+S z+T\right)\left(1-\frac{1}{2} \log z+\ldots\right)
$$

where $M, N,$. etc are written in terms of $S O(12)$ Casimirs (see 47 for details). The discriminant locus that we can realise here will be:

$$
\Delta \sim z^{9}+\mathcal{O}\left(z^{10}\right)
$$

and therefore would show an $E_{7}$ singularity. On the other hand, we won't be able to realise the maximal $S O(12) \times S U(2)$ subgroup here. The curve (4.40) will reflect the following subalgebra:

$$
s u(6) \oplus s u(2) \oplus u(1)
$$

where the $S U(2)$ is the same $S U(2)$ symmetry that gets broken completely to give us an unbroken global $S U(2)$ in (4.37). Also as expected the $\mathbf{1 2}$ and $\mathbf{3 2}$ of $S O(12)$ decomposes as:

$$
12=\mathbf{6}_{+1}+\mathbf{6}_{-1}, \quad 32=\mathbf{1}_{+3}+\mathbf{1}_{-3}+\mathbf{1 5}_{-1}+\overline{\mathbf{1 5}}_{+1}
$$


under $S U(6) \times U(1)$. The monodromy matrix is now different from (4.29) that we had earlier for the $E_{6}$ case. It is given by

$$
\left(\begin{array}{ll}
-2 & -3 \\
-1 & -2
\end{array}\right)
$$

although the same dyonic point is enclosed. The two monodromy matrices (4.29) and (4.44) differ by the monodromy matrix $\left(\begin{array}{ll}1 & 1 \\ 0 & 1\end{array}\right)$ as expected.

As before the manifold (4.37) is not quite the quaternionic manifold that we are looking for. Our aim is to get the non-compact version of this. Therefore using the compact and non-compact generators of $E_{7}$ we can construct the following manifold:

$$
\frac{E_{7(-5)}}{S O(12) \times S U(2)}
$$

which is the required quaternionic manifold falling in the classification of Alekseevskii [3]. In this classification the manifold (4.45) is known as $V(1,4)$ manifold, and should be generated from a Kähler space via the $c$-map. So the question is: can we derive the Kähler space associated with (4.45) using our argument of partial gauging? From the technique developed in earlier sections, we have to look for the $U(1)$ subgroup of the ungauged group in the global symmetry. Here the ungauged group is $S O(12)$ whose subgroup is clearly $S U(6) \times U(1)$. Therefore from the sequencing of the magic square, we can predict the Kähler space to be:

$$
\frac{S O^{*}(12)}{S U(6) \times U(1)}
$$

which when acted by the $c$-map will generate (4.45). The other coset spaces associated with the magic square can also be easily generated using the arguments of the previous sections. The final magic square sequence for $E_{7}$ will be given by:

\begin{tabular}{|c|c|c|c|}
\hline $\mathrm{Sp}(3)$ & $\frac{\mathrm{SU}^{*}(6)}{\mathrm{Sp}(3)}$ & $\frac{\mathrm{SO}^{*}(12)}{\mathrm{SU}(6) \times \mathrm{U}(1)}$ & $\frac{\mathrm{E}_{7(-5)}}{\mathrm{SO}(12) \times \mathrm{SU}(2)}$ \\
\hline
\end{tabular}

which is consistent with the classification [3]. Observe that to go from the second element from the left of the sequence to the third element we use the $r$-map. This is universal for the whole magic square. 


\section{3. $E_{8}$ quaternionic space}

Our next exceptional global symmetry that we want to study here is $E_{8}$. This is straightforward (modulo some subtlety that we mention below) from all the considerations of the previous sections. The extended Dynkin diagram is now given by:

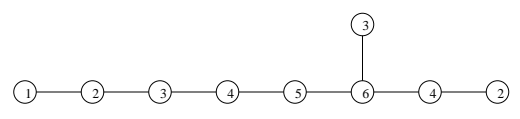

From here the relevant allowed maximal subalgebras are

$$
e_{7} \oplus s u(2), \quad s o(16), \quad s u(5) \oplus s u(5), \quad s u(3) \oplus e_{6}, \quad s u(9)
$$

out of which only two of them, namely, so(16) and $e_{7} \oplus s u(2)$ are also symmetric subalgebras. We can now easily ignore the $S O(16)$ subgroup because we are looking for constrained instantons associated with the $S U(2)$ group. Again constrained instantons exist because $\pi_{3}\left(\frac{E_{8}}{E_{7}}\right)=1$. The 248 of $E_{8}$ then decomposes as 28 :

$$
248=(1,3)+(133,1)+(56,2)
$$

under $E_{7} \times S U(2)$ subgroup. Once we give an expectation value to $(\mathbf{5 6 , 2})$ we can break both the local and global $S U(2)$ s to give us an unbroken global $S U(2)$. Therefore the final symmetry group $E_{7} \times S U(2)$ is completely global and we can now gauge the $S U(2)$ subgroup. Constrained instantons can exist for the $S U(2)$ theory, and they are fibered over the base manifold:

$$
\frac{E_{8}}{E_{7} \times S U(2)}
$$

which gives us the quaternionic Kähler manifold associated with $E_{8}$ global symmetry.

There are few other details we could consider parallel to the details associated with other $E_{n}$ groups studied above. First is the existence of Seiberg-Witten curve for $E_{8}$ global

28 Observe that $\mathbf{2 4 8}$ is the dimension of the adjoint representation of $E_{8}$. This is the smallest representation of $E_{8}$. There is no smaller fundamental representation. This would mean - from our earlier analysis of the potential in (3.1) - we can no longer use the argument of the quaternion $q$ being in fundamental of the global $E_{8}$. However since there is no simple lagrangian formulation of this theory, an absence of fundamental representation may not pose an issue in constructing the vacuum manifold. Indeed as we will show below, there is a possible alternative way to verify that the moduli space of these instantons do not change when we work with the adjoint representation of $E_{8}$. We will deal with this issue in more details in the sequel to this paper. 
symmetry that could be described here by deforming the $E_{7}$ curve (4.40). This deformation is simple and is explained in [47]. The curve therefore is:

$$
\tilde{y}^{2}=\tilde{x}^{3}-\left(z^{2} T_{2}+\mathcal{O}\left(z^{2}\right)\right) \tilde{x}-\left[2 z^{5}+z^{4}\left(T_{6}+\frac{T_{2} T_{4}}{6}+\ldots\right)+\mathcal{O}\left(z^{3}\right)\right]
$$

where $T_{i}$ are $S O(16)$ Casimirs. For more details the readers can refer to [47. The manifest subalgebra that one gets from analysing the curve is neither $s o(16)$ not $e_{7} \oplus s u(2)$ rather it is:

$$
s u(7) \oplus s u(2) \oplus u(1)
$$

which in turn means that the breaking pattern of $E_{7}$ global symmetry is not directly to (4.51) but through an intermediate $s u(8)$ subalgebra. In terms of the corresponding groups this is:

$$
E_{7} \quad \rightarrow \quad S U(8) \rightarrow S U(7) \times U(1)
$$

under which 56 and 133 should be decomposed. The associated monodromy matrix containing the same dyonic point is:

$$
\left(\begin{array}{ll}
-3 & -5 \\
-1 & -2
\end{array}\right)
$$

under the decomposition (4.52). Using this monodromy matrix one can construct the other generators of $E_{7}$ non-perturbatively.

As before the quaternionic manifold of interest is not quite (4.49). We have to look for the non-compact version of this. This is given by:

$$
\frac{E_{8(-24)}}{E_{7} \times S U(2)}
$$

and is known as $V(1,8)$ manifold in the classification of Alekseevskii [3]. The associated Kähler manifold should have the necessary $U(1)$ coset as predicted in (4.5). Gauging the $U(1)$ will correspond to the semilocal strings. The Kähler manifold therefore is:

$$
\frac{E_{7(-25)}}{E_{6} \times U(1)}
$$

which under $c$-map will reproduce (4.54). Similarly (4.55) should come from the $r$-map of a real coset space according to (4.5). The final sequence therefore should be: 


\begin{tabular}{|l|c|c|c|}
\hline $\mathrm{F}_{4}$ & $\frac{\mathrm{E}_{6(-26)}}{\mathrm{F}_{4}}$ & $\frac{\mathrm{E}_{7(-25)}}{\mathrm{E}_{6} \times \mathrm{U}(1)}$ & $\frac{\mathrm{E}_{8(-24)}}{\mathrm{E}_{7} \times \mathrm{SU}(2)}$ \\
\hline
\end{tabular}

which is again consistent with the existing classification [3]. In addition to the above scheme, observe that the generators of the $E_{n}$ exceptional groups appearing in the magic square can be alternatively formulated in the following way [45]:

$$
\begin{aligned}
& E_{6}=S O(8)+S U(3)+6 \times 7=28+8+6 \times 7=78 \\
& E_{7}=S O(8)+S p(3)+12 \times 7=28+21+12 \times 7=133 \\
& E_{8}=S O(8)+F_{4}+24 \times 7=28+52+24 \times 7=248
\end{aligned}
$$

where the existence of $S O(8)=\operatorname{Spin}(8)$ has to do with the underlying triality symmetry 455 and the Lie groups in (4.56) are precisely the $F_{4}, C_{3}$ and $A_{2}$ groups appearing in the magic square.

Finally, before ending this section, let us come back to the issue of $E_{8}$ representation that we discussed briefly at the beginning. An alternative way to verify that we have the correct one-instanton moduli space is to use the adjoint hypermultiplets of $\mathcal{N}=2$ gauge theory. The $E_{8}$ global symmetry can be enhanced to $E_{8}$ gauge symmetry by changing the Seiberg-Witten curve (4.50) to a new one. The curve for this case takes the following general form [59]:

$$
y+\frac{\mu^{2}}{y}+\mathcal{P}_{\mathcal{R}}\left(x ; u_{j}\right)=0
$$

where $\mathcal{P}_{\mathcal{R}}$ is a polynomial in $x$ of order $\operatorname{dim}(\mathcal{R})$, and $\mathcal{R}$ is the adjoint representation of $E_{8}$; $\tilde{y}$ in (4.50) and $y$ differ at most by the polynomial $\mathcal{P}_{\mathcal{R}}$. The other terms occuring in (4.57) are defined as follows: $\mu \equiv \Lambda^{h}$ where $h$ is the dual Coxeter number of $E_{8}$ and $\Lambda$ is the Pauli-Villars scale. The functions $u_{j}, j=1,2, \ldots 8$ are the fundamental Casimirs of $E_{8}$ with the top Casimir $u_{8}$ has degree $h$. By changing (4.50) to (4.57) we have actually enhanced the susy to $\mathcal{N}=4$. Now it is well known that for $E_{8}$ small instantons in $\mathcal{N}=4$ gauge theory the moduli space is indeed given by (4.49), thus confirming our above analysis.

\section{4. $F_{4}$ quaternionic space}

The final example of exceptional global symmetry is $F_{4}$ whose properties are not very different from all the other $E_{n}$ examples that we have been studying so far. In fact $F_{4}$ symmetry is very close to the exceptional $E_{6}$ symmetry. One hint comes from the folding relation between the Dynkin diagrams of $E_{6}$ and $F_{4}$ : 


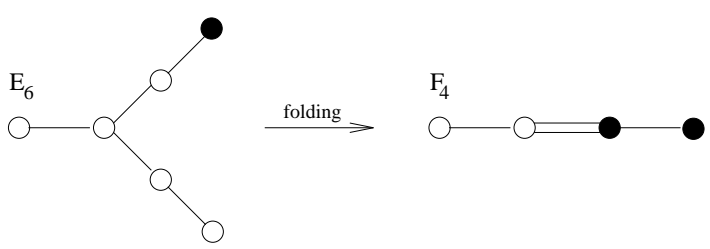

Such similarity between the Dynkin diagrams is also reflected in the corresponding SeibergWitten curves near $z=0$ point. The curves for $F_{4}$ and $E_{6}$ have the following structures:

\begin{tabular}{|c|c|c|c|c|c|}
\hline group & $k(z)$ & $l(z)$ & $h(z)$ & $f(z)$ & $g(z)$ \\
\hline$F_{4}$ & $z^{2}$ & $z$ & $z^{2}$ & $z^{3}$ & $z^{4}$ \\
\hline$E_{6}$ & $z^{2}$ & $z$ & $z^{2}$ & $z^{3}$ & $z^{5}$ \\
\hline
\end{tabular}

where we have referred to only the highest order polynomials for a given coefficient. Clearly the singularity structures of both the curves would then be very similar. Indeed the discriminant of $F_{4}$ curve is given by:

$$
\Delta \sim z^{8}+\mathcal{O}\left(z^{9}\right)
$$

which is identical to the $E_{6}$ curve (4.11). The distinction between the two curves come from analysing points $z \neq 0$. The fundamental representation of $F_{4}$ is $\mathbf{2 6}$ dimensional whereas the fundamental representation of $E_{6}$ is $\mathbf{2 7}$, so they differ by a singlet. The maximal subalgebras of $F_{4}$ can be extracted from the extended Dynkin diagram of $F_{4}$ :

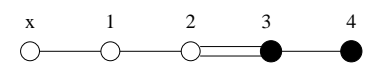

by cutting the diagram at various points. This will give rise to the following subalgebras:

$$
s o(9), \quad s u(3) \oplus s u(3), \quad s u(2), \quad s p(3) \oplus s u(2), \quad g_{2} \oplus s u(2)
$$

out of which we will only keep $s p(3) \oplus s u(2)$ subalgebra because we want to keep the symmetric subgroups. Clearly the group $G_{2} \times S U(2)$ corresponding to the maximal subalgebra $g_{2} \oplus s u(2)$ is not symmetric, and therefore we will not quotient $F_{4}$ by this subgroup. Under $S p(3) \times S U(2)$ subgroup the $\mathbf{2 6}$ of $F_{4}$ decomposes as

$$
26=(6,2)+(14,1)
$$


Giving VEV to $(\mathbf{6 , 2})$ we can break the global and local $S U(2)$ s to have an unbroken $S U(2)$. Since $\pi_{3}\left(\frac{F_{4}}{S p(3)}\right)=1$, the constrained instantons will be fibered over the following quaternionic manifold:

$$
\frac{F_{4}}{S p(3) \times S U(2)}
$$

which is a compact manifold by construction. The manifold that we are concerned about is the non-compact version of (4.61). This is given by:

$$
\frac{F_{4(+4)}}{S p(3) \times S U(2)}
$$

which is also known as $V(1,1)$ manifold in the classification of Alekseevskii [3]. The $S p(3)$ part of the subgroup $S p(3) \times S U(2)$ used for quotienting $F_{4}$ is ungauged. To construct the relevant Kähler manifold associated with (4.62) we need the symmetric subgroup of $S p(3)$. From [38] we see that there is one unique subgroup: $S U(3) \times U(1) \equiv U(3)$ containing a $U(1)$. This means that for a theory with $S p(3)$ global symmetry semilocal strings can exist by gauging the $U(1)$ subgroup. This immediately gives us the corresponding Kähler manifold associated with (4.62):

$$
\frac{S p(3, \mathbf{R})}{S U(3) \times U(1)}
$$

from which (4.62) can be generated by a $c$-map. The real manifold associated with (4.63) can be similarly constructed by looking into the symmetric subgroup of $S U(3)$ that doesn't have an $U(1)$ factor. This subgroup is $S O(3)$ [38], and therefore the magic square sequence for $F_{4}$ symmetry will be:

\begin{tabular}{|c|c|c|c|}
\hline $\mathrm{SO}(3)$ & $\frac{\mathrm{SL}(3, \mathrm{R})}{\mathrm{SO}(3)}$ & $\frac{\mathrm{Sp}(3, \mathrm{R})}{\mathrm{U}(3)}$ & $\frac{\mathrm{F}_{4(+4)}}{\mathrm{Sp}(3) \times \mathrm{SU}(2)}$ \\
\hline
\end{tabular}

where $S L(3, \mathbf{R})$ is the non-compact group associated with the compact group $S U(3)$. The Kähler manifold (4.62) is the real image of the second coset from the left of the magic square. It is also interesting to note that the $\mathbf{5 2}$ of $F_{4}$ can be connected to spin $(8) \equiv S O(8)$ in the following way:

$$
F_{4}=S O(8)+S O(3)+3 \times 7=28+3+3 \times 7=52
$$

which is much like (4.56) described earlier. Finally, to determine the sigma-model description of the quaternionic manifold (4.61) or (4.62) we will need the $F$ function that describes the metric of the Kähler manifold (4.63). This will be determined in subsection 4.6 . 


\subsection{Other examples of quaternionic spaces}

After describing the complete magic square in terms of constrained instantons and possible other semilocal solitons, let us now use the same procedure to study other coset spaces in string theory.

\section{Example 1: U(p) local symmetry and $\mathbf{S U}(\mathbf{n}+\mathbf{p})$ global symmetry}

Our first example is for a $U(p)$ gauge theory with a global symmetry $S U(n+p)$. The extended Dynkin diagram for such a symmetry is

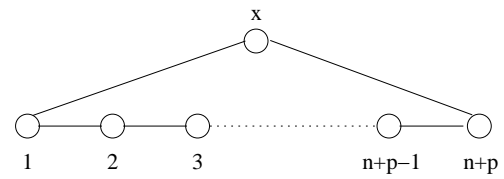

which will give us a symmetric subgroup of $S U(n) \times S U(p) \times U(1)$ 38. The existence of the extra $U(1)$ factor commuting with $S U(n)$ group can be directly explained from the corresponding gauge theory dynamics (see [34] for details).

The above theory can also be realised in the Seiberg-Witten setup by slightly modifying the present scenario. First of all we need a genus $g=p-1$ curve instead of genus one curves that we have been studying so far. The construction of such a curve is very well known [60] so we will be brief. The curve for $\mathcal{N}=2 U(p)$ gauge theory with $S U(n+p)$ global symmetry is [60]:

$$
y^{2}=\left[x^{p}+\sum_{i=2}^{p} s_{i} x^{p-i}+\Lambda^{p-n} \sum_{i=0}^{n} g_{i} x^{n-i}\right]^{2}-\Lambda^{p-n} x^{n+p}
$$

where $\Lambda$ is the Pauli-Villars scale and $\left(s_{i}, g_{i}\right)$ are some constants that depend on the parameters of the theory. The exponent of $\Lambda$ is evaluated as:

$$
\Lambda^{\mathcal{I}\left(\mathbf{R}_{A}\right)-\mathcal{I}\left(\mathbf{R}_{M}\right)}
$$

where $\mathcal{I}\left(\mathbf{R}_{A}\right), \mathcal{I}\left(\mathbf{R}_{M}\right)$ are the Dynkin indices of the adjoint representations of vector multiplet and representations of matter hypermultiplets respectively [61].

The vacuum manifold of this theory will be a Stiefel manifold $\mathbf{V}_{n+p, p}$ [25] which is a space of $p$-frames in $\mathbf{C}^{n+p}$. This is isomorphic to $\frac{S U(n+p)}{S U(n)}$. At low energy the sigma model target space therefore will be given by the following manifold:

$$
\mathbb{C} G(n, p) \equiv \frac{S U(n+p)}{S U(n) \times S U(p) \times U(1)}
$$


which is nothing but the manifold constructed by modding out $U(p)$ gauge orbits from the Stiefel manifold. This immediately implies:

$$
\mathbf{V}_{n+p, p} \approx U(p) \otimes_{f} \mathbb{C} G(n, p)
$$

where the subscript $f$ implies non-trivial fibration. Thus the Stiefel manifold is a $U(p)$ bundle over a Grassmanian manifold. The quaternionic extension of the above case is to consider the complex Grassman manifold $\mathbb{C} G(n, 2)$. This is denoted as $\mathbf{G r}_{2}\left(\mathbf{C}^{n+2}\right)$ in (1.1). For our purpose, however, we need the non-compact version of this space. This is given by:

$$
\frac{S U(n, 2)}{S U(n) \times S U(2) \times U(1)}
$$

The constrained instantons will be non-trivially fibered over (4.69) in the theory. The manifold (4.69) can be mapped to the corresponding Kähler space by gauging a $U(1)$ subgroup of the unbroken group. The Kähler space corresponding to (4.69) is:

$$
\frac{U(n-1,1)}{U(n-1) \times U(1)}
$$

where (4.70) and (4.69) are related by a $c$-map as expected. Observe that the unbroken subgroup in $(4.70)$ is $U(n-1) \equiv S U(n-1) \times U(1)$. To get the corresponding real manifold - that could be related to (4.70) by an inverse $r$-map - we need a subgroup of $U(n-1)$ that doesn't have a $S U(2)$ or an $U(1)$ factor. This is not possible, so our simple rule tells us that there could be no non-zero dimensional real space associated with (4.70). This can be confirmed (see for example [23]). The sequence therefore is:

\begin{tabular}{|l|l|l|l|}
\hline 0 & 0 & $\frac{\mathrm{U}(\mathrm{n}-1,1)}{\mathrm{U}(\mathrm{n}-1) \times \mathrm{U}(1)}$ & $\frac{\mathrm{SU}(\mathrm{n}, 2)}{\mathrm{SU}(\mathrm{n}) \times \mathrm{U}(2)}$ \\
\hline
\end{tabular}

which fits into Alekseevskii classification [3] as well as the recent completion [23]. Notice that for $n=1$ there is no Kähler space.

\section{Example 2: SU(2) local symmetry and $\mathrm{SO}(\mathrm{p}+\mathrm{q})$ global symmetry}

Our next example is almost self-explanatory. This is a $S U(2)$ Seiberg-Witten theory with $S O(p+q)$ global symmetry. The symmetric subgroup of $S O(p+q)$ from any of the two extended Dynkin diagrams (related to $B_{n}$ and $D_{n}$ ): 

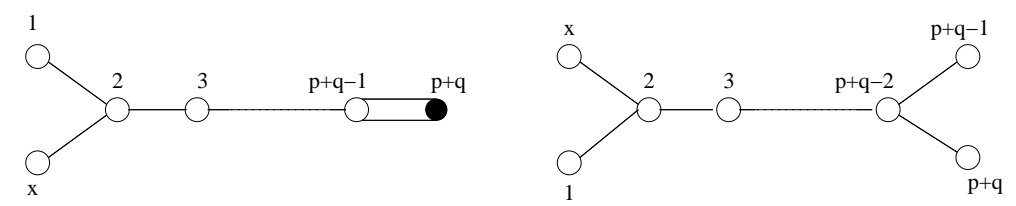

is $S O(p) \times S O(q)$. Therefore taking $S O(7)$ global symmetry, or more appropriately, $S O(3,4)$ global symmetry we can easily find constrained instantons in the theory that are fibered over the following quaternionic space:

$$
\frac{S O(3,4)}{S U(2) \times S U(2) \times S U(2)}
$$

The steps to generate Kähler space associated with semilocal strings is also evident: we have to mod the non-compact version of $S O(4)$ global symmetry by $U(1) \times U(1)$ symmetry. The manifold therefore is

$$
\left[\frac{S U(1,1)}{U(1)}\right]^{2}
$$

so that gauging one of the $U(1)$ we can get semilocal strings in our theory. Manifolds (4.72) and (4.71) are related by a $c$-map. The real manifold associated with (4.72) is clearly $S O(1,1)$. The sequence therefore is:

\begin{tabular}{|l|l|l|l|}
\hline 0 & $\mathrm{SO}(1,1)$ & {$\left[\frac{\mathrm{SU}(1,1)}{\mathrm{U}(1)}\right]^{2}$} & $\frac{\mathrm{SO}(3,4)}{[\mathrm{SU}(2)]^{3}}$ \\
\hline
\end{tabular}

which fits consistently with the de Wit-Van Proeyen completion [23] of Alekseevskii's classification [3].

Let us consider one more example that is in the same vein as our previous example. For this case we take $p=q=4$ so that our non-compact global symmetry is $S O(4,4)$. Clearly the maximal (and symmetric) subgroup is $S O(4) \times S O(4) \equiv[S U(2)]^{4}$, so that the constrained instantons are fibered over the following quaternionic manifold:

$$
\frac{S O(4,4)}{S O(4) \times S O(4)}
$$

where we have, as usual, gauged a $S U(2)$ subgroup of the maximal group. The ungauged subgroup therefore is $S O(4) \times S U(2) \equiv[S U(2)]^{3}$ whose non-compact version would be $[S U(1,1)]^{3}$. To determine the Kähler manifold we have to gauge an $U(1)$ subgroup of $[S U(1,1)]^{3}$ so that we are studying semilocal strings. The Kähler manifold will have more or less the same coset structure as (4.72) discussed above because the ungauged subgroups 
are of the same form as above. Following this trend, the sequence of manifolds that we now expect are:

\begin{tabular}{|l|l|l|l|}
\hline 0 & {$[\mathrm{SO}(1,1)]^{2}$} & {$\left[\frac{\mathrm{SU}(1,1)}{\mathrm{U}(1)}\right]^{3}$} & $\frac{\mathrm{SO}(4,4)}{\mathrm{SO}(4) \times \mathrm{SO}(4)}$ \\
\hline
\end{tabular}

which again fits perfectly with the classification of [23]. The zero dimensional manifold in the last box of the sequence is expected because the real manifold doesn't have a coset structure. In fact so long as $p \leq 4, q \leq 4$ we don't expect to get a non-zero dimensional manifold. This should give us a hint that if we choose a more generic global symmetry from the start, then maybe we could get a non-trivial manifold in the last box of the corresponding sequence. This is indeed the case if we choose $p=P+4, q=4$ with $P$ any integer. The sequence of manifolds are rather straightforward to determine and they are of the following form:

\begin{tabular}{|l|l|l|l|}
\hline $\mathrm{SO}(\mathrm{P}, 1)$ & $\frac{\mathrm{SO}(\mathrm{P}+1,1)}{\mathrm{SO}(\mathrm{P}+1)} \times \mathrm{SO}(1,1)$ & $\frac{\mathrm{SU}(1,1)}{\mathrm{U}(1)} \times \frac{\mathrm{SO}(\mathrm{P}+2,2)}{\mathrm{SO}(\mathrm{P}+2) \times \mathrm{SO}(2)}$ & $\frac{\mathrm{SO}(\mathrm{P}+4,4)}{\mathrm{SO}(\mathrm{P}+4) \times \mathrm{SO}(4)}$ \\
\hline
\end{tabular}

where we see that we do get a manifold in the last box from which we can get the real, Kähler and quaternionic manifolds by various possible mappings. Needless to say, the above sequence fits with the classifications of [3], [23].

\section{Example 3: New sequence of Kahler manifolds in the magic square}

Our final example is a rather curious one. Let us look at the third row of the magic square containing the elements associated with $E_{7}$ etc.:

\begin{tabular}{|c|c|c|c|}
\hline $\operatorname{Sp}(3, R)$ & $\mathrm{SU}(3,3)$ & $\mathrm{SO} *(12)$ & $\mathrm{E}_{7(-25)}$ \\
\hline$S U(3) \times U(1)$ & $\operatorname{SU}(3) \times \mathrm{U}(3)$ & $\mathrm{SU}(6) \times \mathrm{U}(1)$ & $\mathrm{E}_{6} \times \mathrm{U}(1)$ \\
\hline
\end{tabular}

By construction these are all Kähler manifolds that are related to the corresponding semilocal strings (observe the $U(1)$ quotients). An inverse $r$-map to each of these cosets will give us the corresponding real manifolds that we studied in the earlier sections. For example for the unbroken $E_{6}$ subgroup of (4.55) has the following symmetric subgroups:

$$
F_{4}, \quad S U(6) \times S U(2), \quad S O(10) \times U(1), \quad S p(4)
$$

out of which we have used $F_{4}$ to construct the real manifold $\frac{E_{6(-26)}}{F_{4}}$. The other subgroup $S U(6) \times S U(2)$ was used in a different example to construct a quaternionic manifold 
(which is of course unrelated to this sequence of magic square). So we can ask the following question: what if instead of (4.55) we want to construct coset space associated with $S O(10) \times U(1)$ symmetry? This would mean that we are again looking for semilocal strings for a $U(1)$ gauge theory with $E_{6}$ global symmetry. For such a case the associated coset space will be:

$$
\frac{E_{6(-14)}}{S O(10) \times U(1)}
$$

which was first conjectured by [4. Here we see that there is a natural way to justify 29 the existence of such coset space! But this is not the end of the story. Let us look at the next element in the above row of the magic square. The symmetric subgroups of $S U(6)$ are:

$$
S p(3), \quad S U(4) \times S U(2) \times U(1), \quad S U(4), \quad S U(3) \times U(3)
$$

where $S p(3)$ was used earlier to build a real space $\frac{S U^{*}(6)}{S p(3)}$ whereas $S U(3) \times U(3)$ was used in a different sequence of the magic square to construct a Kähler manifold (4.32). Out of the remaining ones we can build a new non-compact coset space:

$$
\frac{S U(4,2)}{S U(4) \times S U(2) \times U(1)}
$$

which in fact does exist in supergravity literature as target space of some sigma model of $\mathcal{N}=2$ supergravity. Thus a new sequence, not realised directly in the magic square, will be:

\begin{tabular}{|c|c|c|c|}
\hline$\frac{\mathrm{SU}(2,1)}{\mathrm{U}(2)}$ & $\frac{\mathrm{SU}(2,1) \times \mathrm{SU}(2,1)}{\mathrm{SU}(2) \times \mathrm{U}(2)}$ & $\frac{\mathrm{SU}(4,2)}{\mathrm{SU}(4) \times \mathrm{U}(2)}$ & $\frac{\mathrm{E}_{6(-14)}}{\mathrm{SO}(10) \times \mathrm{U}(1)}$ \\
\hline
\end{tabular}

which could in principle be embedded in the magic square using the Rozenfeld-Tits constructions [7]. For some more details about these $U(1)$ quotients the readers may want to look up [26].

29 One can view the coset (4.75) as a plane in the sense of projective geometry. The elements of this plane belong to certain Jordan pair such that one can define points and lines along with an incidence relation among them. It turns out that the group $E_{6(-14)}$ acts transitively on points and the stability group of a fixed point is $S O(10) \times U(1)$, thus realising the correspondence between the plane and the coset space (4.75) (see [62] for details). 


\subsection{A note on holomorphic F-functions}

In the previous subsections we discussed the issue of $F$-functions that could be used to determine the metric on the quaternionic Kähler manifolds. In this section we will complete the analysis by postulating the procedure to determine the $F$-function for any given Kähler manifolds. Although the following analysis is standard (see for example [5], [23]) the $F$-functions for $E_{n}$ and $F_{4}$ cases have not been explicitly presented anywhere 30 .

Throughout this section, we use the canonical parametrization introduced by [23] and and the third reference of [16] but where all indices are shifted by one unit in order to fit our notation. The indices $A, B, C=2, \ldots \ldots, n+1$ have been decomposed into indices $2,3, \mu$ and $m$, where $\mu$ and $m$ take respectively $q+1$ and $r$ values.

From [23], we know the form of the cubic functions $C(h)$ in terms of scalar fields $h^{A}$ associated to the real manifolds of rank 1 and 2 :

$$
\begin{aligned}
C(h)= & d_{A B C} h^{A} h^{B} h^{C}=\left(h^{2}\right)^{3}-\frac{1}{2} h^{2}\left(h^{\alpha}\right)^{2}+ \\
& +\frac{1}{\sqrt{2}}\left\{\left(h^{3}\right)^{3}-3 h^{3}\left(\left(h^{\mu}\right)^{2}-\frac{1}{2}\left(h^{m}\right)^{2}\right)+\frac{3}{2} \sqrt{3}\left(\gamma_{\mu}\right)_{m n} h^{\mu} h^{m} h^{n}\right\}
\end{aligned}
$$

with $\alpha \in\{3, \ldots, n+1\}$ and where the gamma matrices $\left(\gamma_{\mu}\right)_{m n}$ are viewed as $r \times r$ matrices generating a $(q+1)$-dimensional Clifford algebra denoted $\mathbf{C}(q+1,0)$.

The coefficients $d_{A B C}$ can also be used to describe Kähler manifold 31 . By imposing the following conditions on the symmetric tensor $d_{A B C}$ [23]:

$$
d_{333}=\frac{1}{\sqrt{2}}, \quad d_{3 \mu \mu}=d_{3 m m}=0, \quad d_{\mu m m}=0
$$

we construct the holomorphic functions $F\left(X^{I}\right)$, in terms of complex variables $X^{I}$, associated to Kähler manifolds that are in the image of an r-map:

$$
\begin{aligned}
F\left(X^{I}\right) & =i d_{A B C} \frac{X^{A} X^{B} X^{C}}{X^{1}} \\
& =\frac{i}{X^{1}}\left\{\left(X^{2}\right)^{3}-\frac{1}{2} X^{2}\left(X^{\alpha}\right)^{2}+\frac{1}{\sqrt{2}}\left(X^{3}\right)^{3}+3\left(\gamma_{\mu}\right)_{m n} X^{\mu} X^{m} X^{n}\right\}
\end{aligned}
$$

30 See however equations (3.38) to (3.42) in the recent paper [63]. We thank Sergio Ferrara for pointing this to us. It will be interesting to relate these values to the ones that we determine here.

31 Note that the use of the canonical parametrisation defines the tensor $d_{A B C}$ up to arbitrary $O(n-1)$ rotations. 
As explained in the third reference of [16], these conditions constrain the allowed values of $q$ to $1,2,4$ and 8 . Since $r=2 q$ and $n=3(q+1)$ for Kähler manifolds, these are exactly the spaces corresponding to the magic square with $n=6,9,15,27$ [4].

\begin{tabular}{|c|c|c|c|c|c|}
\hline $\mathrm{X}(\mathrm{P}, \mathrm{q})$ & Real & $\mathrm{H}(\mathrm{P}, \mathrm{q})$ & Kahler & $\mathrm{V}(\mathrm{P}, \mathrm{q})$ & Quaternionic \\
\hline \hline $\mathrm{X}(1,1)$ & $\mathrm{SL}(3, \mathrm{R}) / \mathrm{SO}(3)$ & $\mathrm{H}(1,1)$ & $\mathrm{Sp}(3) / \mathrm{U}(3)$ & $\mathrm{V}(1,1)$ & $\mathrm{F}_{4} / \mathrm{Sp}(3) \times \mathrm{SU}(2)$ \\
\hline $\mathrm{X}(1,2)$ & $\mathrm{SL}(3, \mathrm{C}) / \mathrm{SU}(3)$ & $\mathrm{H}(1,2)$ & $\mathrm{SU}(3,3) / \mathrm{SU}(3) \times \mathrm{SU}(3) \times \mathrm{U}(1)$ & $\mathrm{V}(1,2)$ & $\mathrm{E}_{6} / \mathrm{SU}(6) \times \mathrm{SU}(2)$ \\
\hline $\mathrm{X}(1,4)$ & $\mathrm{SU}^{*}(6) / \mathrm{Sp}(3)$ & $\mathrm{H}(1,4)$ & $\mathrm{SO} *(12) / \mathrm{SU}(6) \times \mathrm{U}(1)$ & $\mathrm{V}(1,4)$ & $\mathrm{E}_{7} / \mathrm{SO}(12) \times \mathrm{SU}(2)$ \\
\hline $\mathrm{X}(1,8)$ & $\mathrm{E}_{6} / \mathrm{F}_{4}$ & $\mathrm{H}(1,8)$ & $\mathrm{E}_{7} / \mathrm{E}_{6} \times \mathrm{U}(1)$ & $\mathrm{V}(1,8)$ & $\mathrm{E}_{8} / \mathrm{E}_{7} \times \mathrm{SU}(2)$ \\
\hline
\end{tabular}

Table 1: Rank 2 homogeneous special real spaces and their corresponding rank 3 and rank 4 Kahler and Quaternionic spaces respectively associated with the magic square

These Kähler manifolds are respectively associated to the Jordan algebras $J^{3}(\mathbf{R}), J^{3}(\mathbf{C})$, $J^{3}(\mathbf{H})$, and $J^{3}(\mathbf{O})$. They were classified in [5]: the Kähler $H(P, q)$ spaces generate quaternionic $V(P, q)$ spaces [3] under c-map. This in turn emerge from the real $X(P, q)$ manifolds under the r-map [23] (See Table 1 above for a list of relevant coset spaces).

The trivial case $q=0$ with $n=3$, which is also generated by the above restriction, is part of the Kähler $K(P, \dot{P})$ space and is associated with $W(P, \dot{P})$ quaternionic manifolds. $P$ and $\dot{P}$ represents the multiplicity of each irreducible representations of the Clifford Algebras which are listed in Table 2:

\begin{tabular}{|c|c|c|}
\hline $\mathrm{q}$ & $\mathrm{C}(\mathrm{q}+1,0)$ & $\mathrm{Dq}+1$ \\
\hline \hline 1 & $\mathrm{R}(2)$ & 2 \\
\hline 2 & $\mathrm{C}(2)$ & 4 \\
\hline 4 & $\mathrm{H}(2) \times \mathrm{H}(2)$ & 8 \\
\hline 8 & $\mathrm{R}(16) \times\{\mathrm{R} \times \mathrm{R}\}$ & 16 \\
\hline
\end{tabular}

Table 2: $\mathrm{C}(\mathrm{q}+1,0)$ represents real Clifford algebras, $\mathrm{K}(\mathrm{n})$ are $\mathrm{nxn}$ matrices with entries over the field $\mathrm{K}$ and $\mathrm{D}_{\mathrm{q}+1}$ represents the real dimension of an irreducible representation of the Clifford algebra

We restrict our study to $q>0$ cases. In order to classify all $F$-functions associated to $H(P, q)$, one needs to consider all gamma matrices generating a $(q+1)$ dimensional real Clifford algebra with positive metric. This classification was done in [64, see Table 3 below for the relevant cases.

Solutions are characterised by specifying the multiplicities $P$ and $\dot{P}$ of each irreducible representations of the Clifford algebras. In all cases we will discuss, we will consider $\dot{P}=0$ 
and $P=1$. The generating matrices $\sigma_{i}$ used in the above table are simply the Pauli matrices:

$$
\sigma_{1}=\left(\begin{array}{cc}
0 & 1 \\
1 & 0
\end{array}\right), \quad \sigma_{2}=\left(\begin{array}{cc}
0 & -i \\
i & 0
\end{array}\right), \quad \sigma_{3}=\left(\begin{array}{cc}
1 & 0 \\
0 & -1
\end{array}\right)
$$

The $\gamma_{i}$ matrices are the Dirac Gamma matrices made out of the sigma matrices in the standard way. The $\phi_{i}$ matrices are in turn made of the $\gamma_{i}$ matrices in the following way:

$$
\phi_{j}=\left(\begin{array}{cc}
0 & i \gamma_{j} \\
-i \gamma_{j} & 0
\end{array}\right), \quad \phi_{6}=\left(\begin{array}{cc}
\mathbf{I}_{4} & 0 \\
0 & -\mathbf{I}_{4}
\end{array}\right), \quad \phi_{7}=\left(\begin{array}{cc}
0 & \mathbf{I}_{4} \\
\mathbf{I}_{4} & 0
\end{array}\right), \quad j=1,2,3,4,5
$$

Finally the $\varpi_{j}$ are similarly constructed using the $\phi_{j}$ matrices in exactly the same way as above with $j$ running from $j=1,2, \ldots \ldots, 7$. The other two matrices $\varpi_{8}$ and $\varpi_{9}$ are constructed by $\mathbf{I}_{8}$ like $\phi_{6}$ and $\phi_{7}$ respectively.

\begin{tabular}{|c|c|c|}
\hline Algebra & $\begin{array}{c}\text { Matrix } \\
\text { Dimensions }\end{array}$ & $\begin{array}{c}\text { Generating } \\
\text { Matrices }\end{array}$ \\
\hline \hline $\mathrm{C}_{2}$ & $2 \times 2$ & $\mathrm{i} \sigma_{2}, \mathrm{i} \sigma_{3}$ \\
\hline $\mathrm{C}_{3}$ & $2 \times 2$ & $\sigma_{1}, \sigma_{2}, \sigma_{3}$ \\
\hline $\mathrm{C}_{4}$ & $4 \times 4$ & $\gamma_{1}, \gamma_{2}, \gamma_{3}, \gamma_{4}$ \\
\hline $\mathrm{C}_{5}$ & $4 \times 4$ & $\mathrm{i} \gamma_{1}, \mathrm{i} \gamma_{2}, \mathrm{i} \gamma_{3}, \ldots, \mathrm{i} \gamma_{5}$ \\
\hline $\mathrm{C}_{6}$ & $8 \times 8$ & $\mathrm{i} \phi_{\mathrm{j}}, \mathrm{j}=1,2,3, \ldots \ldots, 6$ \\
\hline $\mathrm{C}_{7}$ & $8 \times 8$ & $\phi_{\mathrm{j}}, \mathrm{j}=1,2,3, \ldots \ldots ., 7$ \\
\hline $\mathrm{C}_{8}$ & $16 \times 16$ & $\varpi_{\mathrm{j}}, \mathrm{j}=1,2,3, \ldots \ldots ., 8$ \\
\hline $\mathrm{C}_{9}$ & $16 \times 16$ & $\mathrm{i} \overline{\mathrm{j}}_{\mathrm{j}}, \mathrm{j}=1,2,3, \ldots . ., 9$ \\
\hline
\end{tabular}

Table 3: Classification of generating matrices of Clifford Algebras

We associate $C_{n}$ with $C_{(q+2)}$. Hence, $C_{3}$ is associated to $\mathbf{R}(2) \in C(q+1,0)_{q=1}, C_{4}$ to $\mathbf{C}(2)$, etc. This association allows us to generate a $(q+1)$-dimensional Clifford Algebra with $r \times r$ basis that satisfy simultaneously the condition imposed in (4.79) on the gamma matrices i.e. $\left(\gamma_{\mu}\right)_{m m}=0$. Thus, say we have $\sigma_{1}, \sigma_{2}, \sigma_{3}$ and we impose the condition $\left(\sigma_{\mu}\right)_{m m}=0$, the term $\left(\sigma_{\mu}\right)_{m n}$ will therefore be equal to zero when it comes to $\sigma_{3}$ and we will be left with two $(2 \times 2)$ matrices i.e. $\sigma_{1}, \sigma_{2}$ to $\operatorname{span} \mathbf{R}(2)$ as required.

We are now ready to construct the $F\left(X^{I}\right)$ holomorphic functions for each Kähler spaces associated to the magic square. For the Kähler space associated with $G_{2}$ coset we already gave the $F$-function in (3.27), and for the coset associated with $S p(n+1)$ we know that there is no Kähler space (see section 3.1 for details). 
- Kähler space $H(1,1)$ :

For $H(1,1), q=1, r=2$ and $n=6$. Hence $A, B, C=2, \ldots \ldots, 7$. In addition, $\mu \in\{4, \ldots \ldots, 7\}$ and takes exactly $q+1$ values say $\mu=4,5$ whereas $m \in\{4, \ldots \ldots, 7\}$ and takes $r$ values for instance $m=5,6$. The quantity $\alpha$ takes all values in $\{3, \ldots ., 7\}$. The matrices generating the Clifford algebra $\mathbf{R}(2) \in \mathbf{C}_{3}$ would be $\sigma_{1}, \sigma_{2}$ according to the previous argument and we shall rename them $\sigma_{\mu}$. Furthermore $\mathcal{F}\left(X^{I}\right) \equiv-i X^{1} F\left(X^{I}\right)$ :

$$
\begin{aligned}
\mathcal{F}\left(X^{I}\right)=\left(X^{2}\right)^{3}- & \frac{1}{2} X^{2}\left(X^{3}\right)^{2}-\ldots . .-\frac{1}{2} X^{2}\left(X^{7}\right)^{2}+\frac{1}{\sqrt{2}}\left(X^{3}\right)^{3}+ \\
& +3\left(\sigma_{4}\right)_{m n} X^{4} X^{m} X^{n}+3\left(\sigma_{5}\right)_{m n} X^{5} X^{m} X^{n}
\end{aligned}
$$

- Kähler space $H(1,2)$ :

For $H(1,2), q=2, r=4, n=9, \mu \in\{4, \ldots \ldots, 10\}$ and takes 3 values say $4,5,6$ and $m \in\{4, \ldots \ldots, 10\}$ takes 4 values say $7,8,9,10 . \alpha \in\{3, \ldots \ldots, 10\}$ and the Clifford algebra would be generated by $\gamma_{2}, \gamma_{3}, \gamma_{4}$ which we rename $\gamma_{\mu}$ :

$$
\begin{aligned}
\mathcal{F}\left(X^{I}\right)=\left(X^{2}\right)^{3}- & \frac{1}{2} X^{2}\left(X^{3}\right)^{2}-\ldots \ldots-\frac{1}{2} X^{2}\left(X^{10}\right)^{2}+\frac{1}{\sqrt{2}}\left(X^{3}\right)^{3}+ \\
& +3\left(\gamma_{4}\right)_{m n} X^{4} X^{m} X^{n}+3\left(\gamma_{5}\right)_{m n} X^{5} X^{m} X^{n}+3\left(\gamma_{6}\right)_{m n} X^{6} X^{m} X^{n}
\end{aligned}
$$

- Kähler space $H(1,4)$ :

For $H(1,4), q=4, r=8, n=15, \mu \in\{4, \ldots \ldots, 16\}$ and takes 5 values say $4,5,6,7,8$ and $m \in\{4, \ldots \ldots, 16\}$ takes 8 values say $9,10,11,12,13,14,15,16 . \alpha \in\{3, \ldots \ldots, 16\}$ and the Clifford algebra would be generated by five $(8 \times 8)$ elements of $C_{6}$ i.e. $i \phi_{j}$ with $j=1, \ldots \ldots, 5$ which we rename $i \phi_{\mu}$ :

$$
\begin{aligned}
\mathcal{F}\left(X^{I}\right)=\left(X^{2}\right)^{3}- & \frac{1}{2} X^{2}\left(X^{3}\right)^{2}-\ldots \ldots-\frac{1}{2} X^{2}\left(X^{16}\right)^{2}+\frac{1}{\sqrt{2}}\left(X^{3}\right)^{3}+ \\
& +3 i\left(\phi_{4}\right)_{m n} X^{4} X^{m} X^{n}+\ldots \ldots+3 i\left(\phi_{8}\right)_{m n} X^{8} X^{m} X^{n}
\end{aligned}
$$

- Kähler space $H(1,8)$ :

For $H(1,8), q=8, r=16, n=27, \mu \in\{4, \ldots \ldots, 28\}$ and takes 9 values and $m \in\{4, \ldots \ldots, 28\}$ takes 16 values. $\alpha \in\{3, \ldots \ldots, 28\}$ and the Clifford algebra would be generated by nine $(16 \times 16)$ elements of $C_{9}$ i.e. $\varpi_{j}$ with $j=1, \ldots \ldots, 9$ which we rename $\varpi_{\mu}$ :

$$
\begin{aligned}
\mathcal{F}\left(X^{I}\right)=\left(X^{2}\right)^{3}- & \frac{1}{2} X^{2}\left(X^{3}\right)^{2}-\ldots \ldots-\frac{1}{2} X^{2}\left(X^{28}\right)^{2}+\frac{1}{\sqrt{2}}\left(X^{3}\right)^{3}+ \\
& +3\left(\varpi_{4}\right)_{m n} X^{4} X^{m} X^{n}+\ldots \ldots .+3\left(\varpi_{12}\right)_{m n} X^{12} X^{m} X^{n}
\end{aligned}
$$


The above analysis therefore summarises all the $F$-functions that we need to determine the Kähler spaces. To get the corresponding quaternionic spaces, we use the metric given in (3.29) for each of the four cases. With this therefore we have the complete picture of all the Quaternionic and the Kähler manifolds in the magic square.

\section{Summary, discussions and future directions}

In this paper we hopefully gave a new way to study the magic square in mathematics and string theory that is not based on the dimensional reduction of supergravity theories. Our method relies on the existence of constrained instantons in certain $\mathcal{N}=2$ gauge theories with exceptional global symmetries. These theories are not asymptotically free and are at strong coupling. This means that a simple Yang-Mills description may not suffice and we might even lack a lagrangian description of these theories. Nevertheless we have ample evidence that these theories exist: via Seiberg-Witten curves, F-theory and possible quaternionic formulations of low energy descriptions.

Viewing them as sectors of Seiberg-Witten theories, the exceptional global symmetries form non-trivial fixed points of renormalisation group flows. This is well known and they lead to the following sequence of theories:

$$
E_{8} \longrightarrow E_{7} \longrightarrow E_{6} \longrightarrow D_{4} \longrightarrow A_{2} \longrightarrow A_{1} \longrightarrow\{0\}
$$

Our idea of sequential gauging is partially motivated by the above sequence. The $S U(2)$ constrained instanton which is also a semilocal instanton for our case is constructed by gauging an $S U(2)$ subgroup of the global group. The $U(1)$ part of ungauged global group that also contains the monodromy associated with a dyonic point - is then further gauged to construct semilocal strings in the model. These two process give us Quaternionic and Kähler spaces that are related by a $c$-map. Once we have these spaces, the real space associated to the Kähler space can be easily constructed.

Our whole analysis therefore depends on the existence of one instanton moduli space in these theories. In the absence of a proper lagrangian description we cannot give a concrete construction of these instantons solutions of course, but moduli space can still be constructed. Existence of Seiberg-Witten curves also means that we have added all the 
instanton contributions in the path-integral. Recall that the instanton contributions to the Seiberg-Witten prepotential $\mathcal{F}_{\mathrm{SW}}$ can be written as:

$$
\mathcal{F}_{\text {SW }}=\mathcal{F}_{\text {classical }}+\mathcal{F}_{\text {one-loop }}+\frac{1}{2 \pi i} \sum_{k=1}^{\infty} \int_{\mathcal{M}_{k}} \omega e^{-S} \Lambda^{k\left(4-N_{F}\right)}
$$

where $\mathcal{M}_{k}$ is the moduli space of $k$-instantons, $\omega$ is the volume form, $S$ is the instanton action, $N_{F}$ is the number of flavors and $\Lambda$ is the same Pauli-Villars scale that we used earlier. It is therefore an interesting question to ask how instantons in these gauge theories with $E_{n}, F_{4}$ global symmetries give us the right Seiberg-Witten curves. Note however that if one breaks the $E_{n}$ symmetry by giving masses to quarks and keeping the gauge coupling finite, one may hope to get a convergent expression for the instanton partition function. However, to show that an analytic continuation to the $E_{n}$ symmetric point would make sense, requires more work 32 . We leave this aspect for future work.

Another interesting direction is to look for theories with exceptional gauge symmetries. Incidentally one instanton moduli spaces will be the same for these theories - its just an embedding of $S U(2)$ in exceptional gauge groups 33 - but the corresponding curves will be different. We gave one example before. Another example would be a theory with $F_{4}$ gauge symmetry. Such a theory with one massless hypermultiplet has the following Seiberg-Witten curve 34 [61:

$$
y^{2}=\left[\left(x^{2}-b_{1}^{2}\right)\left(x^{2}-b_{2}^{2}\right)\left(x^{2}-b_{3}^{2}\right)\left(x^{2}-b_{4}^{2}\right)\right]^{2}-x^{4} \Lambda^{12}
$$

where $b_{i}$ are the projections of the weights: $(1000),(-1100),(0-111)$ and $(00-11)$. It would be interesting to study these theories with more than one massless hypermultiplets.

One final issue is the classification of de-Wit and Van-Proeyen [23] that completes Alekseevskii's classification of Quaternionic manifolds [3]. We have shown that we can reproduce all of Alekseevskii's symmetric manifolds and few more of de-Wit and VanProeyen also. However we haven't investigated enough to see whether we could reproduce all other manifolds in the classification of [23]. In fact its an interesting question to ask whether these manifolds have a coset structure like the other manifolds in the classification. We leave this for future work.

32 We thank Nikita Nekrasov for comments on this.

33 Recall $\pi_{3}\left(E_{n}\right)=\pi_{3}\left(F_{4}\right)=\mathbf{Z}$.

34 This could also be derived from (4.57) with appropriate polynomial $\mathcal{P}_{\mathcal{R}}$. 


\section{Acknowledgements}

We would like to thank Rhiannon Gwyn for initial collaboration; Sergio Ferrara, Mark Hindmarsh, Sheldon Katz, Joseph Minahan, Nikita Nekrasov, Savdeep Sethi, Ulrich Theis, David Tong, Tanmay Vachaspati and Alexei Yung for helpful correspondences and especially Ori Ganor, Tom Kephart and V. P Nair for important clarifications and discussions. The work of KD is supported by a NSERC grant. The work of VH is partially supported by a NSERC grant and the work of AW is supported in part by a NSERC grant and in part by the university research grant. 


\section{References}

[1] M.Berger, "Sur les groupes d'holonomie des variétes a connexions affines et des variétés riemannienes," Bull.Soc.Math.France 83 (1955), 279; "Sur les groupes d'holonomie homogenes des variétés riemannienes," C.R. Acad.Sci.Paris serie A262 (1966), 1316.

[2] J. A. Wolf, "Complex homogeneous contact manifolds and quaternionic symmetric spaces," J. of Math. Mech., 14 (1965), 1033.

[3] D.V. Alekseevskii "Riemannian manifolds with exceptional holonomy groups," Funct. Anal. Appl. 2 (1968), 97; "Compact quaternion spaces," Funct. Anal. Appl. 2 (1968), 106; "Classification of quaternionic spaces with transitive solvable group of motions," Math. USSR-Izv. 9 (1975), 297.

[4] M. Gunaydin, G. Sierra and P. K. Townsend, "Exceptional Supergravity Theories And The Magic Square," Phys. Lett. B 133, 72 (1983); "The Geometry Of N=2 MaxwellEinstein Supergravity And Jordan Algebras," Nucl. Phys. B 242, 244 (1984); "Gauging The D $=5$ Maxwell-Einstein Supergravity Theories: More On Jordan Algebras," Nucl. Phys. B 253, 573 (1985).

[5] S. Cecotti, "Homogeneous Kahler manifolds and $\mathrm{T}$ algebras in $\mathrm{N}=2$ supergravity and superstrings," Commun. Math. Phys. 124, 23 (1989); S. Cecotti, S. Ferrara and L. Girardello, "Geometry of Type II Superstrings and the Moduli of Superconformal Field Theories," Int. J. Mod. Phys. A 4, 2475 (1989).

[6] S. Ferrara and S. Sabharwal, "Quaternionic Manifolds for Type II Superstring Vacua of Calabi-Yau Spaces," Nucl. Phys. B 332, 317 (1990); "Dimensional reduction of type II superstrings," Class. Quant. Grav. 6, L77 (1989).

[7] H. Freudenthal, "Beziehungen der $E_{7}$ und $E_{8}$ zur Oktavenebene", I, II, Indag. Math. 16 (1954), 218-230, 363-368. III, IV, Indag. Math. 17 (1955), 151-157, 277-285. VIX, Indag. Math. 21 (1959), 165-201, 447-474. X, XI, Indag. Math. 25 (1963) 457487; B. A. Rosenfeld, "Geometrical interpretation of the compact simple Lie groups of the class $E_{n}$ (Russian)", Dokl. Akad. Nauk. SSSR 106 (1956) 600-603; J. Tits, " Le plan projectif des octaves et les groupes exceptionnels $E_{6}$ et $E_{7}$ ", Bull. Acad. Roy. Belg. Sci. 40 (1954), 29-40.

[8] N. Seiberg and E. Witten, "Electric - magnetic duality, monopole condensation, and confinement in N=2 supersymmetric Yang-Mills theory," Nucl. Phys. B 426, 19 (1994) [Erratum-ibid. B 430, 485 (1994)], hep-th/9407087; "Monopoles, duality and chiral symmetry breaking in $\mathrm{N}=2$ supersymmetric QCD," Nucl. Phys. B 431, 484 (1994), hep-th/9408099.

[9] I. Affleck, "On constrained instantons," Nucl. Phys. B 191, 429 (1981).

[10] A. Achucarro and T. Vachaspati, "Semilocal cosmic strings," Phys. Rev. D 44, 3067 (1991); "Semilocal and electroweak strings," Phys. Rept. 327, 347 (2000), hepph/9904229. 
[11] I. I. Pjateckii-Sapiro, "The structure of j-algebras", Izv. Akad. Nauk SSSR Ser. Mat. 26, 453 (1962); Am. Math. Soc. Transl. 55, 207 (1966); S. G. Gindikin, I. I. PjateckiiSapiro, E. B. Vinberg, "Homogeneous Kahler manifolds" in Geometry of Homogeneous Bounded Domains, pp. 3-87 (1968); E. B. Vinberg, S. G. Gindikin and I. I. PjateckiiSapiro, "Classification and canonical realization of complex homogeneous bounded domains", Trudy. Moskov. Mat. Obshch. 12, 359 (1963).

[12] P. Jordan, "Über eine klasse nichtassoziativer hyperkomplexer algebren," Nachr. Ges. Wiss. Göttingen 569 (1932).

[13] L. Alvarez-Gaume and D. Z. Freedman, "Geometrical Structure And Ultraviolet Finiteness In The Supersymmetric Sigma Model," Commun. Math. Phys. 80, 443 (1981).

[14] B. Zumino, "Supersymmetry And Kahler Manifolds," Phys. Lett. B 87, 203 (1979).

[15] E. Witten and J. Bagger, "Quantization Of Newton's Constant In Certain Supergravity Theories," Phys. Lett. B 115, 202 (1982).

[16] J. Bagger and E. Witten, "Matter Couplings In N=2 Supergravity," Nucl. Phys. B 222, 1 (1983); B. de Wit and A. Van Proeyen, "Potentials And Symmetries Of General Gauged N=2 Supergravity: Yang-Mills Models," Nucl. Phys. B 245, 89 (1984); E. Cremmer, C. Kounnas, A. Van Proeyen, J. P. Derendinger, S. Ferrara, B. de Wit and L. Girardello, "Vector Multiplets Coupled To N=2 Supergravity: Superhiggs Effect, Flat Potentials And Geometric Structure," Nucl. Phys. B 250, 385 (1985).

[17] A. Strominger, "Loop corrections to the universal hypermultiplet," Phys. Lett. B 421, 139 (1998), hep-th/9706195; H. Gunther, C. Herrmann and J. Louis, "Quantum corrections in the hypermultiplet moduli space," Fortsch. Phys. 48, 119 (2000), hepth/9901137; R. Bohm, H. Gunther, C. Herrmann and J. Louis, "Compactification of type IIB string theory on Calabi-Yau threefolds," Nucl. Phys. B 569, 229 (2000), hep-th/9908007.

[18] D. Robles-Llana, F. Saueressig and S. Vandoren, "String loop corrected hypermultiplet moduli spaces," JHEP 0603, 081 (2006), hep-th/0602164.

[19] D. Robles-Llana, M. Rocek, F. Saueressig, U. Theis and S. Vandoren, "Nonperturbative corrections to $4 \mathrm{D}$ string theory effective actions from $\mathrm{SL}(2, \mathrm{Z})$ duality and supersymmetry," Phys. Rev. Lett. 98, 211602 (2007), hep-th/0612027; D. Robles-Llana, F. Saueressig, U. Theis and S. Vandoren, "Membrane instantons from mirror symmetry," 0707.0838 [hep-th].

[20] N. Berkovits and W. Siegel, "Superspace Effective Actions for 4D Compactifications of Heterotic and Type II Superstrings," Nucl. Phys. B 462, 213 (1996), hep-th/9510106; N. Seiberg and E. Witten, "Gauge dynamics and compactification to three dimensions," hep-th/9607163; B. R. Greene, D. R. Morrison and C. Vafa, "A geometric 
realization of confinement," Nucl. Phys. B 481, 513 (1996), hep-th/9608039; I. Antoniadis, S. Ferrara, R. Minasian and K. S. Narain, " $\mathrm{R}^{* *} 4$ couplings in M- and type II theories on Calabi-Yau spaces," Nucl. Phys. B 507, 571 (1997), hep-th/9707013.

[21] I. Antoniadis, R. Minasian, S. Theisen and P. Vanhove, "String loop corrections to the universal hypermultiplet," Class. Quant. Grav. 20, 5079 (2003), hep-th/0307268.

[22] S. V. Ketov, "Summing up D-instantons in N = 2 supergravity," Nucl. Phys. B 649, 365 (2003), hep-th/0209003; "Instanton-induced scalar potential for the universal hypermultiplet," Nucl. Phys. B 656, 63 (2003), hep-th/0212003; "D-instantons and matter hypermultiplet," Phys. Lett. B 558, 119 (2003), hep-th/0302001; L. Anguelova, M. Rocek and S. Vandoren, "Quantum corrections to the universal hypermultiplet and superspace," Phys. Rev. D 70, 066001 (2004), hep-th/0402132; N. Halmagyi, I. V. Melnikov and S. Sethi, "Instantons, Hypermultiplets and the Heterotic String," 0704.3308 [hep-th].

[23] B. de Wit and A. Van Proeyen, "Special geometry, cubic polynomials and homogeneous quaternionic spaces," Commun. Math. Phys. 149, 307 (1992), hep-th/9112027.

[24] P. Chen, K. Dasgupta, K. Narayan, M. Shmakova and M. Zagermann, "Brane inflation, solitons and cosmological solutions: I," JHEP 0509, 009 (2005), hep-th/0501185; K. Dasgupta, H. Firouzjahi and R. Gwyn, "Lumps in the throat," JHEP 0704, 093 (2007), hep-th/0702193.

[25] M. Hindmarsh, R. Holman, T. W. Kephart and T. Vachaspati, "Generalized semilocal theories and higher Hopf maps," Nucl. Phys. B 404, 794 (1993), hep-th/9209088.

[26] K. Higashijima and M. Nitta, "Supersymmetric nonlinear sigma models as gauge theories," Prog. Theor. Phys. 103, 635 (2000), hep-th/9911139; M. Arai and M. Nitta, "Hyper-Kaehler sigma models on (co)tangent bundles with $\mathrm{SO}(\mathrm{n})$ isometry," Nucl. Phys. B 745, 208 (2006), hep-th/0602277; Y. Isozumi, M. Nitta, K. Ohashi and N. Sakai, "Construction of non-Abelian walls and their complete moduli space," Phys. Rev. Lett. 93, 161601 (2004), hep-th/0404198.

[27] K. Dasgupta, J. P. Hsu, R. Kallosh, A. Linde and M. Zagermann, "D3/D7 brane inflation and semilocal strings," JHEP 0408, 030 (2004), hep-th/0405247.

[28] A. Hanany and D. Tong, "Vortices, instantons and branes," JHEP 0307, 037 (2003), hep-th/0306150; "On monopoles and domain walls," Commun. Math. Phys. 266, 647 (2006), hep-th/0507140; M. Shifman and A. Yung, "Non-Abelian semilocal strings in N = 2 supersymmetric QCD," Phys. Rev. D 73, 125012 (2006), hep-th/0603134; M. Eto et al., "On the moduli space of semilocal strings and lumps," 0704.2218 [hep-th].

[29] E. Witten, "Small Instantons in String Theory," Nucl. Phys. B 460, 541 (1996), hepth/9511030.

[30] O. J. Ganor and A. Hanany, "Small $E_{8}$ Instantons and Tensionless Non-critical Strings," Nucl. Phys. B 474, 122 (1996), hep-th/9602120. 
[31] A. Sen, "F-theory and Orientifolds," Nucl. Phys. B 475, 562 (1996), hep-th/9605150; T. Banks, M. R. Douglas and N. Seiberg, "Probing F-theory with branes," Phys. Lett. B 387, 278 (1996), hep-th/9605199.

[32] M. R. Douglas, "Branes within branes," Cargese 1997, Strings, branes and dualities 267 (1995), hep-th/9512077; "Gauge Fields and D-branes," J. Geom. Phys. 28, 255 (1998), hep-th/9604198.

[33] N. Dorey, T. J. Hollowood, V. V. Khoze, M. P. Mattis and S. Vandoren, "Multiinstanton calculus and the AdS/CFT correspondence in $\mathrm{N}=4$ superconformal field theory," Nucl. Phys. B 552, 88 (1999) hep-th/9901128; N. Dorey, T. J. Hollowood, V. V. Khoze and M. P. Mattis, "The calculus of many instantons," Phys. Rept. 371, 231 (2002), hep-th/0206063.

[34] I. Antoniadis and B. Pioline, "Higgs branch, hyperKaehler quotient and duality in SUSY N = 2 Yang-Mills theories," Int. J. Mod. Phys. A 12, 4907 (1997), hepth/9607058; L. Anguelova, M. Rocek and S. Vandoren, "Hyperkaehler cones and orthogonal Wolf spaces," JHEP 0205, 064 (2002), hep-th/0202149; B. de Wit, M. Rocek and S. Vandoren, "Gauging isometries on hyperKaehler cones and quaternion-Kaehler manifolds," Phys. Lett. B 511, 302 (2001), hep-th/0104215; "Hypermultiplets, hyperkaehler cones and quaternion-Kaehler geometry," JHEP 0102, 039 (2001), hepth/0101161; S. Vandoren, "Instantons and quaternions," hep-th/0009150.

[35] A. Swann, "Hyperkähler and quaternionic Kähler geometry", Math. Ann. 289, 421 (1991).

[36] G. 't Hooft, "Computation of the quantum effects due to a four-dimensional pseudoparticle," Phys. Rev. D 14, 3432 (1976) [Erratum-ibid. D 18, 2199 (1978)]; Y. Frishman and S. Yankielowicz, "Large Order Behavior Of Perturbation Theory And Mass Terms," Phys. Rev. D 19, 540 (1979).

[37] A. P. Balachandran, V. P. Nair, N. Panchapakesan and S. G. Rajeev, "Low Mass Solitons From Fractional Charges In QCD," Phys. Rev. D 28, 2830 (1983).

[38] W. J. MacKay and J. Patera, "Tables of Dimensions, Indices, and Branching Rules for Representations of Simple Lie Algebras," Marcel Dekker, New York 1981; R. Slansky, "Group Theory For Unified Model Building," Phys. Rept. 79, 1 (1981).

[39] M. R. Gaberdiel and B. Zwiebach, "Exceptional groups from open strings," Nucl. Phys. B 518, 151 (1998), hep-th/9709013.

[40] M. Rocek, C. Vafa and S. Vandoren, "Quaternion-Kahler spaces, hyperkahler cones, and the c-map," math.dg/0603048.

[41] B. de Wit and A. Van Proeyen, "Special geometry, cubic polynomials and homogeneous quaternionic spaces," Commun. Math. Phys. 149, 307 (1992), hep-th/9112027.

[42] E. Cartan, "Oeuvres complétes", Gauthier-Villars, Paris (1952).

[43] J.E. Humphreys, "Introduction to Lie Algebras and Representation Theory," Springer, NY (1972). 
[44] J. Beckers, V. Hussin and P. Winternitz, "Nonlinear equations with superposition formulas and the exceptional group $G_{2}$. I. Complex and real forms of $g_{2}$ and their maximal subalgebras," Journ. Math. Phys. 27, 2217 (1986); "Nonlinear equations with superposition formulas and the exceptional group $G_{2}$. II. Classification of the equations," Journ. Math. Phys. 28, 520 (1987).

[45] G. M. Dixon, "Division algebras: octonions, quaternions, complex numbers and the algebraic design of physics," Kluwer Academic Publishers, 1994.

[46] J. M. Landsberg and L. Manivel, "The projective geometry of Freudenthal's magic square," math.AG/9908039; A. Sudbery, "Division algebras," J. Phys. A: Math. Gen. 17 (1984) 939; E. J. Lohmus, E. Paal, L. Sorgsepp, "Nonassociative algebras in physics," Hadronic Press (1994).

[47] J. A. Minahan and D. Nemeschansky, "An $\mathrm{N}=2$ superconformal fixed point with E(6) global symmetry," Nucl. Phys. B 482, 142 (1996), hep-th/9608047; "Superconformal fixed points with E(n) global symmetry," Nucl. Phys. B 489, 24 (1997), hepth/9610076.

[48] A. Sen, "F-theory and Orientifolds," Nucl. Phys. B 475, 562 (1996), hep-th/9605150.

[49] K. Dasgupta and S. Mukhi, "F-theory at constant coupling," Phys. Lett. B 385, 125 (1996), hep-th/9606044.

[50] W. Lerche and N. P. Warner, "Polytopes And Solitons In Integrable, N=2 Supersymmetric Landau-Ginzburg Theories," Nucl. Phys. B 358, 571 (1991).

[51] N. Seiberg, "IR dynamics on branes and space-time geometry," Phys. Lett. B 384, 81 (1996), hep-th/9606017.

[52] K. Dasgupta, C. Herdeiro, S. Hirano and R. Kallosh, "D3/D7 inflationary model and M-theory," Phys. Rev. D 65, 126002 (2002), hep-th/0203019.

[53] E. Witten, "Solutions of four-dimensional field theories via M-theory," Nucl. Phys. B 500, 3 (1997), hep-th/9703166.

[54] A. Hanany and E. Witten, "Type IIB superstrings, BPS monopoles, and threedimensional gauge dynamics," Nucl. Phys. B 492, 152 (1997), hep-th/9611230.

[55] O. Aharony, A. Hanany and B. Kol, "Webs of (p,q) 5-branes, five dimensional field theories and grid diagrams," JHEP 9801, 002 (1998), hep-th/9710116.

[56] K. Dasgupta and S. Mukhi, "BPS nature of 3-string junctions," Phys. Lett. B 423, 261 (1998), hep-th/9711094.

[57] A. Sen, "String network," JHEP 9803, 005 (1998), hep-th/9711130.

[58] J. A. Bryan, S. M. Carroll and T. Pyne, "A Texture bestiary," Phys. Rev. D 50, 2806 (1994), hep-ph/9312254.

[59] E. J. Martinec and N. P. Warner, "Integrable systems and supersymmetric gauge theory," Nucl. Phys. B 459, 97 (1996), hep-th/9509161; A. Klemm, W. Lerche, P. Mayr, C. Vafa and N. P. Warner, "Self-Dual Strings and N=2 Supersymmetric Field Theory," Nucl. Phys. B 477, 746 (1996), hep-th/9604034; W. Lerche and N. P. Warner, 
"Exceptional SW geometry from ALE fibrations," Phys. Lett. B 423, 79 (1998), hepth/9608183.

[60] A. Klemm, W. Lerche, S. Yankielowicz and S. Theisen, "Simple singularities and N=2 supersymmetric Yang-Mills theory," Phys. Lett. B 344, 169 (1995), hep-th/9411048; 'On The Monodromies Of N=2 Supersymmetric Yang-Mills Theory," hep-th/9412158; P. C. Argyres, M. R. Plesser and A. D. Shapere, "The Coulomb phase of N=2 supersymmetric QCD," Phys. Rev. Lett. 75, 1699 (1995), hep-th/9505100; A. Hanany and $\mathrm{Y}$. Oz, "On the quantum moduli space of vacua of $\mathrm{N}=2$ supersymmetric $\mathrm{SU}(\mathrm{N}(\mathrm{c})$ ) gauge theories," Nucl. Phys. B 452, 283 (1995), hep-th/9505075.

[61] U. H. Danielsson and B. Sundborg, "Exceptional Equivalences in N=2 Supersymmetric Yang-Mills Theory," Phys. Lett. B 370, 83 (1996), hep-th/9511180.

[62] P. Truini, G. Olivieri and L. C. Biedenharn, "The Jordan Pair Content Of The Magic Square And The Geometry Of The Scalars in N=2 Supergravity," Lett. Math. Phys. 9, 255 (1985).

[63] S. Ferrara, E. G. Gimon and R. Kallosh, "Magic supergravities, N = 8 and black hole composites," Phys. Rev. D 74, 125018 (2006), hep-th/0606211.

[64] C. P. Poole, Jr., and H. A. Farach, "Pauli-Dirac Matrix Generators of Clifford. Algebras," Found. Phys. 12, 719 (1982); D. Li, C. P. Poole and H. A. Farach, "A general method of generating and classifying Clifford algebras," J. Math. Phys. 27, 1173 (1986). 